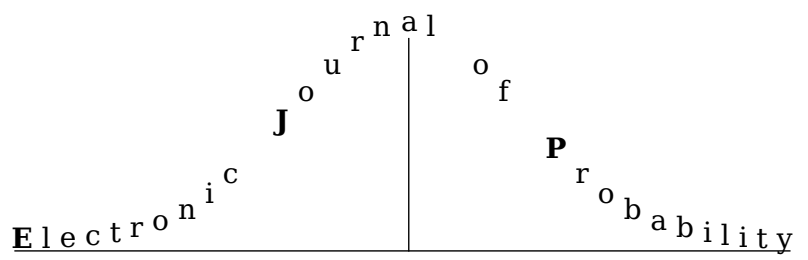

Electron. J. Probab. 24 (2019), no. 102, 1-36.

ISSN: 1083-6489 https://doi.org/10.1214/19-EJP346

\title{
Convergence of Brownian motions on metric measure spaces under Riemannian Curvature-Dimension conditions
}

\author{
Kohei Suzuki*
}

\begin{abstract}
We show that the pointed measured Gromov convergence of the underlying spaces implies (or under some condition, is equivalent to) the weak convergence of Brownian motions under Riemannian Curvature-Dimension conditions.
\end{abstract}

Keywords: Riemannian Curvature-Dimension condition; measured Gromov-Hausdorff convergence; Brownian motion; weak convergence.

AMS MSC 2010: Primary 60F17, Secondary 53C23.

Submitted to EJP on February 1, 2018, final version accepted on July 23, 2019.

\section{Contents}

1 Introduction $\quad 2$

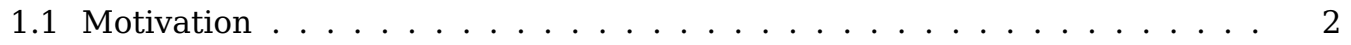

1.2 Main results . . . . . . . . . . . . . . . . . . . . 3

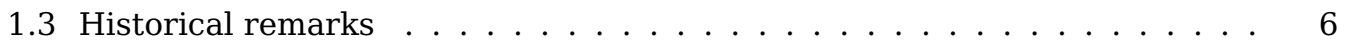

1.4 Organization of the paper ................... 7

2 Notation \& preliminary results $\quad 8$

2.1 Notation . . . . . . . . . . . . . . . . . . . 8

$2.2 L^{p}$-Wasserstein space . . . . . . . . . . . . . . . 8

2.3 Pointed measured Gromov convergence . . . . . . . . . . . . . . 9

2.4 RCD spaces . . . . . . . . . . . . . . . . . . . . . 9

2.4 .1 Relative entropy . . . . . . . . . . . . . . . . . . . . . . . . . . . . . 9

2.4 .2 Cheeger energy . . . . . . . . . . . . . . . . . . . . . 10

$2.4 .3 \operatorname{RCD}(K, \infty)$ spaces . . . . . . . . . . . . . . . . . 10

2.4.4 $W_{2}$-gradient flow of relative entropy . . . . . . . . . . . . . 11

$2.4 .5 L^{2}$-gradient flow of Cheeger energy . . . . . . . . . . . . . . . . 11

$2.4 .6 \operatorname{RCD}^{*}(K, N)$ spaces . . . . . . . . . . . . . . . 12

$2.5 L^{2}$-convergence of heat semigroups under the PmG convergence $\ldots . . .13$

${ }^{*}$ Scuola Normale Superiore. E-mail: kohei.suzuki@sns.it 
3 Brownian motion on RCD spaces 13

3.1 Brownian motions on $\operatorname{RCD}(K, \infty)$ spaces . . . . . . . . . . . 13

3.2 Brownian motions on $\operatorname{RCD}^{*}(K, N)$ spaces $\ldots \ldots \ldots \ldots \ldots$

4 Examples $\quad 16$

4.1 Weighted Riemanniam manifolds and pmG limit spaces . . . . . . . . . 16

4.2 Alexandrov spaces . . . . . . . . . . . . . . . . 17

4.3 Hilbert space with $\log$-concave measures $\ldots \ldots \ldots$. . . . . . . . 18

5 Proof of Theorem $1.2 \quad 20$

6 Proof of Theorem 1.4 28

$\begin{array}{lll}7 & \text { Proof of Theorem 1.6 } & 30\end{array}$

8 Proof of Theorem $1.7 \quad 31$

$\begin{array}{ll}\text { References } & 33\end{array}$

\section{Introduction}

\subsection{Motivation}

The aim of this paper is to characterize a probabilistic convergence of Brownian motions in terms of a geometric convergence of the underlying spaces. Our main results show that the pointed measured Gromov (pmG) convergence of the underlying spaces implies (or under some condition, is equivalent to) the weak convergence of Brownian motions under Riemannian Curvature-Dimension (RCD) conditions for the underlying spaces.

Let us consider the following motivating example: let a sequence of Riemannian manifolds $\left\{M_{n}\right\}_{n \in \mathbb{N}}$ converges to a (possibly non-smooth) metric measure space in the Gromov-Hausdorff (GH) sense. Let $\left(B^{n}, \mathbb{P}^{n}\right)$ be a Brownian motions on each $M_{n}$. Noting that $\left(B^{n}, \mathbb{P}^{n}\right)$ can be determined only by the underlying geometric structure of the Riemannian manifolds $M_{n}$, an important question is whether

(Q) a sequence of Brownian motions on Riemannian manifolds also converges weakly to the Brownian motion on the GH-limit space.

This question however does not make sense without additional assumptions because there is a gap between the geometric and probabilistic convergences: the weak convergence of Brownian motions clearly involves the first-order differentiable structure of the underlying spaces although the GH convergence never sees any information of differentiable structures. Indeed, we have examples whereby the limit process is no more a diffusion process (see (ii) and (iii) in Remark 1.9).

In this paper, adopting as an additional assumption the uniform lower Ricci curvature bound of $M_{n}$, we can answer (Q) affirmatively, which is an application of the main results in this paper. To be more precise, we obtain the equivalence between these geometric/probabilistic convergences in the framework of metric measure spaces under the synthetic lower Ricci curvature bound (called RCD in this paper).

Let us explain the background issues in more detail. Generally, the GH-limit spaces of Riemannian manifolds with lower Ricci curvature bounds (called Ricci limit spaces) are so singular that they are not necessarily even topological manifolds and may have a dense singular set (see Example 4.3). However, they still have "Riemannian-like" structures and similar properties to smooth Riemannian manifolds with lower Ricci curvature bounds, which have been investigated initially by Cheeger-Colding $[17,18,19]$. The 
RCD condition is a proper generalization of the notion of lower Ricci curvature bounds to non-smooth spaces including Ricci limit spaces (see [6, 3, 8, 29, 22]). It is known that RCD spaces include various finite- and infinite-dimensional non-smooth spaces, not only Ricci limit spaces, but also infinite-dimensional spaces such as Hilbert spaces with log-concave measures (related to various stochastic partial differential equations) (see further details in Section 4).

By recent developments of analysis on metric measure spaces, we can construct Brownian motions on RCD spaces by using a certain quadratic form, what is called Cheeger energy. This is a generalization of Dirichlet energy on smooth manifolds and induces a quasi-regular strongly local conservative symmetric Dirichlet form (AmbrosioGigli-Savaré [5, 6], Ambrosio-Gigli-Mondino-Rajala [3]), which is determined only by the underlying metric measure structure.

One of the important problems for Brownian motions on these non-smooth spaces is to characterize the weak convergence of Brownian motions in terms of some geometrical convergence of the underlying spaces, which we call the stability of Brownian motions. The significance of the stability can be explained from several different perspectives. From the standpoint of limit theorems of stochastic processes, the stability is interpreted as a geometric characterization of invariance principles for Brownian motions in the sense that Brownian motions on limit spaces are approximated by Brownian motions on converging spaces. From the viewpoint of "well-definedness", the stability also enables us to verfiy that Brownian motions in limit spaces are "well-defined" in the sense that Brownian motions intrinsically defined by Cheeger energies on limit spaces coincide with limit processes of Brownian motions on approximating spaces. From the perspective that Brownian motions are considered as "a map" assigning laws of diffusions (i.e., probability measures on path spaces) to each metric measure space, the stability reveals the interesting fact that this map is continuous with respect to the corresponding topologies (e.g., GH-topology of metric measure spaces/weak topology of probability measures on path spaces), which is one ideal aspect of Brownian motions but has not been focused on so much until now.

The main contribution of this paper is to prove the stability of Brownian motions in the general framework of RCD spaces, whereby various singular/infinite-dimensional spaces are included. Moreover, we show several equivalences of the weak convergence of Brownian motions and the pmG convergence of the underlying spaces. For references to other investigations regarding the stability problem, see the historical remarks (Section 1.3 below).

\subsection{Main results}

In this paper, we always consider pointed metric measure (p.m.m.) spaces $\mathcal{X}=$ $(X, d, m, \bar{x})$ whereby

$(X, d)$ is a complete separable geodesic metric space with nonnegative and nonzero Borel measure $m$ which is finite on all bounded sets, and $\bar{x}$ is a fixed point in $\operatorname{supp}[m]$.

For main theorems, we assume the following condition:

Assumption 1.1. Let $K \in \mathbb{R}$ and $\overline{\mathbb{N}}:=\mathbb{N} \cup\{\infty\}$. Let $\left\{\mathcal{X}_{n}\right\}_{n \in \overline{\mathbb{N}}}=\left\{\left(X_{n}, d_{n}, m_{n}, \bar{x}_{n}\right)\right\}_{n \in \overline{\mathbb{N}}}$ be a sequence of p.m.m. spaces satisfying (1.1) and $\operatorname{RCD}(K, \infty)$ condition.

The notion of $\operatorname{CD}(K, \infty)$ spaces was introduced by Sturm [65] and Lott-Villani [47], and the notion of $\operatorname{RCD}(K, \infty)$ spaces was introduced by Ambrosio-Gigli-Savaré [6] and Ambrosio-Gigli-Mondino-Rajala [3]. The $\operatorname{CD}(K, \infty)$ condition is a generalization of Ricci curvature bounded from below by $K$ to metric measure spaces in terms of 
the $K$-convexity of the entropy on the Wasserstein spaces. Furthermore $\operatorname{RCD}(K, \infty)$ condition means the $\mathrm{CD}(K, \infty)$ and that the Cheeger energy is quadratic. We will explain the precise definition in Subsection 2.4. RCD spaces admit the GH limit spaces of Riemannian manifolds with lower Ricci curvature bounds, and also admit Alexandrov spaces (metric spaces satisfying a generalized notion of "sectional curvature $\geq K$ ") (Petrunin [55] and Zhang-Zhu [71]), cone spaces and warped product spaces (Ketterer $[39,40]$ ), and quotient spaces (Galaz-García-Kell-Mondino-Sosa [28]). Moreover, not only finite-dimensional spaces, but also several infinite-dimensional spaces related to stochastic partial differential equations are included such as Hilbert spaces with logconcave measures (Ambrosio-Savaré-Zambotti [9]).

Under Assumption 1.1, we can always take constants $c_{1}, c_{2}>0$ satisfying the following volume growth estimate (see [65, Theorem 4.24])

$$
m_{n}\left(B_{r}\left(\bar{x}_{n}\right)\right) \leq c_{1} e^{c_{2} r^{2}}, \quad \forall r>0 .
$$

Here we mean $B_{r}\left(\bar{x}_{n}\right):=\left\{x \in X_{n}: d\left(x, \bar{x}_{n}\right)<r\right\}$. Taking $\mathrm{C}>c_{2}$, we set a weighted measure $\widetilde{m}_{n}$ as follows:

$$
z_{n}:=\int_{X_{n}} e^{-C d_{n}^{2}\left(x, \bar{x}_{n}\right)} d m_{n}(x), \quad \text { and } \quad \widetilde{m}_{n}:= \begin{cases}\frac{1}{z_{n}} e^{-C d_{n}^{2}\left(\cdot, \bar{x}_{n}\right)} m_{n}, & \text { if } m_{n}\left(X_{n}\right)=\infty, \\ \frac{1}{m_{n}\left(X_{n}\right)} m_{n}, & \text { if } m_{n}\left(X_{n}\right)<\infty .\end{cases}
$$

Under Assumption 1.1, the Cheeger energy $\mathrm{Ch}_{n}$ on $\mathcal{X}_{n}=\left(X_{n}, d_{n}, m_{n}, \bar{x}_{n}\right)$ (see Subsection 2.4.2) induces a quasi-regular conservative symmetric strongly local Dirichlet form, and there exists a conservative symmetric Markov process $\left(\left\{\mathbb{P}_{n}^{x}\right\}_{x \in X_{n}},\left\{B_{t}^{n}\right\}_{t \geq 0}\right)$ on $\mathcal{X}_{n}$ (See Section 3.1). We call $\left(\left\{\mathbb{P}_{n}^{x}\right\}_{x \in X_{n}},\left\{B_{t}^{n}\right\}_{t \geq 0}\right)$ Brownian motion on $\mathcal{X}_{n}$.

The following main theorem states that the weak convergence of the Brownian motions can be characterized by the pmG convergence of the underlying spaces under Assumption 1.1 (we will give the definition of the pmG convergence in Subsection 2.3).

Theorem 1.2. Suppose that Assumption 1.1 holds. Then the following (i) and (ii) are equivalent:

\section{(i) (pmG Convergence of the Underlying Spaces)}

The p.m.m. spaces $\left\{\mathcal{X}_{n}\right\}_{n \in \mathbb{N}}$ converge to $\mathcal{X}_{\infty}=\left(X_{\infty}, d_{\infty}, m_{\infty}, \bar{x}_{\infty}\right)$ in the pmG sense.

(ii) (Weak Convergence of the Laws of Brownian Motions)

There exist a complete separable metric space $(X, d)$ and isometric embeddings $\iota_{n}: X_{n} \rightarrow X(n \in \overline{\mathbb{N}})$ so that $\iota_{n}\left(\bar{x}_{n}\right) \rightarrow \iota_{\infty}\left(\bar{x}_{\infty}\right)$, and

$$
\left(\iota_{n}\left(B^{n}\right), \mathbb{P}_{n}^{\widetilde{m}_{n}}\right) \rightarrow\left(\iota_{\infty}\left(B^{\infty}\right), \mathbb{P}_{\infty}^{\widetilde{m}_{\infty}}\right) \quad \text { weakly } \quad \text { in } \mathcal{P}(C([0, \infty) ; X)) .
$$

Here $\left(\iota_{n}\left(B^{n}\right), \mathbb{P}_{n}^{\widetilde{m}_{n}}\right)$ means the law of the embedded Brownian motion $\iota_{n}\left(B^{n}\right)$ with the initial distribution $\widetilde{m}_{n}$ and $\mathcal{P}(C([0, \infty) ; X))$ denotes the set of all Borel probability measures on the continuous path space $C([0, \infty) ; X)$.

Remark 1.3. Several remarks for Theorem 1.2 are given below.

(i) The $\operatorname{RCD}(K, \infty)$ condition is stable under the pmG convergence (see [30, Theorem 7.2]), and therefore the limit space $\mathcal{X}_{\infty}$ also satisfies the $\operatorname{RCD}(K, \infty)$ condition so that the Brownian motion can be defined also on the limit space $\mathcal{X}_{\infty}$.

(ii) The pmG convergence is weaker than the measured Gromov-Hausdorff convergence. See [30, Theorem 3.30]. 
In statement (ii) in Theorem 1.2, the initial distribution is absolutely continuous with respect to the reference measure $m_{n}$. It is natural in the next step to ask how the case of the dirac measure $\delta_{\bar{x}_{n}}$ is, which means the Brownian motions start at the point $\bar{x}_{n}$. We introduce several conditions below:

(A) For any $n \in \mathbb{N}, m_{n}\left(X_{n}\right)=1$.

(B) For any $r>0$ and any $t>0$,

$$
\sup _{n \in \mathbb{N}}\left\|p_{n}\left(t, \bar{x}_{n}, \cdot\right)\right\|_{\infty, B_{r}\left(\bar{x}_{n}\right)}<\infty
$$

whereby $p_{n}(t, x, y)$ is the density of the transition probability $p_{n}(t, x, d y)$ of $\left\{P_{t}^{n}\right\}_{t \geq 0}$ with respect to the reference measure $m_{n}$, and $\|\cdot\|_{\infty, B_{r}\left(\bar{x}_{n}\right)}$ means the essential supremum on the ball $B_{r}\left(\bar{x}_{n}\right)$.

Now we state the second main result.

Theorem 1.4. Suppose that Assumption 1.1 holds. If, moreover, either (A), or (B) holds, then (i) (thus also (ii)) in Theorem 1.2 implies the following (iii) $)_{\varepsilon}$ : for any $\varepsilon>0$,

$\begin{aligned} & \text { (iii) } \geq \varepsilon \text { (Weak Convergence of the Laws of Brownian Motions Starting at Points in a Time } \\ & \text { Interval }[\varepsilon, \infty) \text { ) }\end{aligned}$

There exist a complete separable metric space $(X, d)$ and isometric embeddings $\iota_{n}: X_{n} \rightarrow X(n \in \overline{\mathbb{N}})$ so that $\iota_{n}\left(\bar{x}_{n}\right) \rightarrow \iota_{\infty}\left(\bar{x}_{\infty}\right)$ and

$$
\left(\iota_{n}\left(B^{n}\right), \mathbb{P}_{n}^{\bar{x}_{n}}\right) \rightarrow\left(\iota_{\infty}\left(B^{\infty}\right), \mathbb{P}_{\infty}^{\bar{x}_{\infty}}\right) \quad \text { weakly in } \mathcal{P}(C([\varepsilon, \infty) ; X)) .
$$

Remark 1.5. Several remarks for Theorem 1.4 are given below.

(i) Condition (B) is satisfied for any $\operatorname{RCD}^{*}(K, N)$ spaces according to the Gaussian heat kernel estimate by Jiang-Li-Zhang [35].

(ii) If the following uniform ultra-contractivity of the heat semigroup $\left\{H_{t}\right\}_{t \geq 0}$ holds, then condition (B) holds (see [6, Proposition 6.4]): there exists a $p>1$ so that, with some positive constant $C(t, K)$ dependent only on $t$ and $K$, we have

$$
\left\|H_{t} f\right\|_{p} \leq C(t, K)\|f\|_{1}, \quad \forall f \in L^{1}(X, m), \forall t>0 .
$$

We have examples satisfying the ultra-contractivity which is a $\operatorname{RCD}(K, \infty)$ space but not a $\operatorname{RCD}^{*}(K, N)$ space for any $1<N<\infty$. Let $\mathcal{X}_{\alpha}=\left(\mathbb{R},|\cdot-\cdot|, C_{\alpha} \exp \left\{-|\cdot|{ }^{\alpha}\right\} d x\right)$ whereby $\alpha \in\{2,4,6, \ldots\}$ is an even number and $C_{\alpha}$ is the normalizing constant. For any $\alpha>2$, it is known that $\mathcal{X}_{\alpha}$ satisfies the ultra-contractivity of the heat semigroup (Kavian-Kerkyacharian-Roynette [38]) and satisfies the $\operatorname{RCD}(0, \infty)$ condition, but not $\operatorname{RCD}^{*}(K, N)$ for any finite $1<N<\infty$.

The notion of $\operatorname{RCD}^{*}(K, N)$ condition is a generalization of Ricci curvature bounded from below by $K$ and dimension bounded above by $N$ to metric measure spaces, which is stronger than the $\operatorname{RCD}(K, \infty)$ condition (see $[29,22,8]$ ).

Next we consider the converse implication that the weak convergence of Brownian motions induces the pmG convergence of the underlying spaces. Define $p_{n}(t, x, x)=$ $\left\|p_{n}(t / 2, x, \cdot) p_{n}(t / 2, x, \cdot)\right\|_{2}^{2}$, which can be defined for every $x \in X$. Let us consider the following condition: there exists $t_{*}>0$ and a constant $M$ so that

$$
\sup _{n \in \mathbb{N}} p_{n}\left(t_{*}, \bar{x}_{n}, \bar{x}_{n}\right)<M<\infty .
$$

Note that, since $p_{n}(t, x, x)$ is non-increasing function in $t$, if we find the time $t_{*}$ satisfying (1.5), then for any $t>t_{*}$, the estimate (1.5) holds. We also note that, for fixed $1<N<\infty$ 
and $K \in \mathbb{R}$, if $X_{n}$ satisfies the $\operatorname{RCD}^{*}(K, N)$ for all $n \in \mathbb{N}$ and $\inf _{n} m_{n}\left(B_{1}\left(\bar{x}_{n}\right)\right)>0$ (this holds under (i), (ii), (iii) $\geq_{\geq \varepsilon}$, or (iii) $\geq_{0}$ since $\left\{\iota_{n}\left(\bar{x}_{n}\right)\right\}_{n \in \mathbb{N}}$ is bounded in a common ambient space $X$ according to $\left.\iota\left(\bar{x}_{n}\right) \rightarrow \iota_{\infty}\left(\bar{x}_{\infty}\right)\right)$, then (1.5) is satisfied for some constant $M$ and $t_{*}$ because of the local Gaussian heat kernel estimate by Jiang-Li-Zhang [35]. Let $\operatorname{diam}\left(X_{n}\right)$ denote the diameter of $X_{n}: \operatorname{diam}\left(X_{n}\right):=\sup _{x, y \in X_{n}} d_{n}(x, y)$. We now state the following theorem:

Theorem 1.6. Suppose that Assumption 1.1 and condition (1.5) hold. If, moreover, either $K>0$, or $\sup _{n \in \mathbb{N}} \operatorname{diam}\left(X_{n}\right)<D$ holds for some $0<D<\infty$, then (iii) $\geq \varepsilon$ for any $\varepsilon>0$ in Theorem 1.4 implies (i) and (ii) in Theorem 1.2 (therefore all statements (i), (ii) and (iii) $\geq \varepsilon$ for any $\varepsilon>0$ are equivalent).

Finally, we give the following statement, in which all statements (i), (ii), (iii) $>_{\varepsilon}$ for any $\varepsilon>0$, and (iii) $\geq 0$ are equivalent under the $\operatorname{RCD}^{*}(K, N)$ condition with a uniform diameter bound.

Theorem 1.7. Let $K \in \mathbb{R}, 1<N<\infty$ and $0<D<\infty$. Suppose that a sequence of p.m.m. spaces $\left\{\mathcal{X}_{n}\right\}_{n \in \mathbb{N}}$ satisfies (1.1), $\operatorname{RCD}^{*}(K, N)$ and $\sup _{n \in \mathbb{N}} \operatorname{diam}\left(X_{n}\right)<D$. Then all four statements of (i), (ii) in Theorem 1.2, (iii) $\geq_{\varepsilon}$ for any $\varepsilon>0$ in Theorem 1.4 and the following $(i i i)_{\geq 0}$ are equivalent:

(iii) $\geq 0$ (Weak Convergence of the Laws of Brownian Motions Starting at Points in a Time Interval $[0, \infty)$ )

There exist a compact metric space $(X, d)$ and isometric embeddings $\iota_{n}: X_{n} \rightarrow$ $X(n \in \overline{\mathbb{N}})$ so that

$$
\left(\iota_{n}\left(B^{n}\right), \mathbb{P}_{n}^{\bar{x}_{n}}\right) \rightarrow\left(\iota_{\infty}\left(B^{\infty}\right), \mathbb{P}_{\infty}^{\bar{x}_{\infty}}\right) \quad \text { weakly } \quad \text { in } \mathcal{P}(C([0, \infty) ; X)) .
$$

Remark 1.8. We give several remarks for Theorem 1.7.

(i) The $\operatorname{RCD}^{*}(K, N)$ condition is stable under the pmG convergence (see [22]), and therefore the limit space $\mathcal{X}_{\infty}$ also satisfies the $\operatorname{RCD}^{*}(K, N)$ condition so that the Brownian motion can be defined at every starting point also on the limit space $\mathcal{X}_{\infty}$.

(ii) The pmG convergence (see Definition 2.1) is equivalent to the pointed measured Gromov-Hausdorff convergence under the assumption in Theorem 1.7 (see [30, Theorem 3.33]).

\subsection{Historical remarks}

Remark 1.9. Several historical remarks are given below.

(i) In Ambrosio-Savaré-Zambotti [9, Theorem 1.5], they investigated the weak convergence of Brownian motions on a fixed Hilbert space (as an ambient space) with varying log-concave measures and norms, which is a specific case of $\operatorname{RCD}(0, \infty)$ spaces. Their metrics $d_{n}$ are not necessarily isometric to the metric $d$ in the ambient space, but each $d_{n}$ is equivalent to $d$. In the case that each $d_{n}$ is isometric to $d$, our results (Theorem 1.2 and 1.4) can be seen as a generalization of their result [9, Theorem 1.5] to general $\operatorname{RCD}(K, \infty)$ spaces.

(ii) In Ogura [51], under the condition of uniform upper bounds for heat kernels (not necessarily lower bound of Ricci curvatures) and the Kasue-Kumura (KK) spectral convergence, he studied the weak convergence of the laws of time-discretized Brownian motions on weighted compact Riemannian manifolds. The KK spectral convergence roughly means a uniform convergence of heat kernels and stronger than the mGH convergence. In his case, the Ricci curvature is not necessarily bounded from below and the limit process may be a jump process $([51,4.6])$. 
The time-discretization is one possible approach for a convergence of stochastic processes on varying spaces, while we adopt in this paper a different approach, i.e., embedding into one common metric space $X$.

(iii) If we do not assume RCD conditions for a sequence of the underlying metric measure spaces, then limit processes are not necessarily diffusions. In OguraTomisaki-Tuchiya [52], they considered a sequence of Euclidean spaces $\left(\mathbb{R}^{d},\|\cdot\|_{2}\right)$ with certain underlying measures $\mu_{n}$ whereby $\left\{\left(\mathbb{R}^{d},\|\cdot\|_{2}, \mu_{n}\right)\right\}_{n \in \mathbb{N}}$ do not necessarily satisfy RCD conditions. They showed that diffusion processes on $\mathbb{R}^{d}$ associated with the corresponding local Dirichlet forms converge to jump processes (or generally jump-diffusion processes) corresponding to certain non-local Dirichlet forms.

(iv) In Freidlin-Wentzell [25, 26] and Albeverio-Kusuoka [1] (see also references therein), diffusion processes associated with SDEs on thin tubes in $\mathbb{R}^{d}$ were studied. When thin tubes shrink to a spider graph, diffusion processes converge weakly to a one-dimensional diffusion on this spider graph. Their setting does not satisfy the RCD condition since spider graphs branch at points of conjunctions but RCD spaces are essentially non-branching (see [58, Theorem 1.1]).

(v) In Athreya-Löhr-Winter [10], the weak convergence of certain Markov processes on tree-like spaces was studied. When tree-like spaces converge in Gromov-vague sense, the corresponding processes also converge weakly. Their tree-like spaces admit 0-hyporbolic spaces, which are not necessarily included in RCD spaces.

(vi) In Suzuki [68], the author investigated the weak convergence of continuous stochastic processes on metric spaces converging in the Lipschitz distance. The Lipschitz convergence is stronger than the measured Gromov convergence (see [32, Section 3.C]).

Finally we list related studies not mentioned in Remark 1.9. In Suzuki [69], the author studied the weak convergence of non-symmetric diffusion processes on RCD spaces as a next step of the current paper. In Li $[44,45]$, she studied a convergence of random ODE/SDE on manifolds. In Stroock-Varadhan [60], Stroock-Zheng [61] and Burdzy-Chen [16], approximations of diffusion processes on $\mathbb{R}^{d}$ by discrete Markov chains on $(1 / n) \mathbb{Z}^{d}$ were investigated. In Bass-Kumagai-Uemura [12] and Chen-Kim-Kumagai [20], they studied approximations of jump processes on proper metric spaces by Markov chains on discrete graphs. Approximations of Markov processes on ultra-metric spaces were explored in Suzuki [67]. In Pinsky [56], he studied approximations of Brownian motions on Riemannian manifolds by random walks, while the case of sub-Riemannian manifolds was investigated by Gordina and Laetsch [31]. In Croydon-Hambly-Kumagai [21], in which it was assumed that a sequence of resistance forms converges with respect to the GH-vague topology and satisfies a uniform volume doubling condition, they showed the weak convergence of corresponding Brownian motions and local times. There are many studies about scaling limits of random processes on random environments (see, e.g., Kumagai [41] and references therein).

\subsection{Organization of the paper}

The paper is structured as follows: First, the notation is fixed and preliminary facts are recalled in Section 2 (no new results are included), namely: basic notations and basic definitions (Subsection 2.1); $L^{2}$-Wasserstein distance (Subsection 2.2); pmG convergence (Subsection 2.3); $\operatorname{RCD}(K, \infty)$ and $\operatorname{RCD}^{*}(K, N)$ spaces (Subsection 2.4); $L^{2}$-convergence of the heat semigroup (Subsection 2.5). In Section 3, we state several properties about Brownian motions on RCD spaces. In Section 4 we present examples in which Assumptions 1.1 and the assumption in Theorem 1.7 are satisfied. These examples consist of weighted Riemannian manifolds and their pmG limit spaces, Alexandrov spaces, 
and Hlibert spaces with log-concave probability measures. In Section 5, we give the proof of Theorem 1.2. In Section 6, we show the proof of Theorem 1.4. In Section 7, we prove Theorem 1.6. Finally, in Section 8, we prove Theorem 1.7.

\section{Notation \& preliminary results}

\subsection{Notation}

Let $\mathbb{N}=\{0,1,2, \ldots\}$ and $\overline{\mathbb{N}}:=\mathbb{N} \cup\{\infty\}$ denote the set of natural numbers and the set of natural numbers with $\{\infty\}$ respectively. For a complete separable metric space $(X, d)$, we denote by $B_{r}(x)=\{y \in X: d(x, y)<r\}$ the open ball centered at $x \in X$ with radius $r>0$. By using $\mathscr{B}(X)$, we mean the family of all Borel sets in $(X, d)$; and by $\mathcal{B}_{b}(X)$, the set of real-valued bounded Borel-measurable functions on $X$. Let $C(X)$ be the set of real-valued continuous functions on $X$, while $C_{b}(X), C_{\infty}(X), C_{0}(X)$ and $C_{b s}(X)$ denote the subsets of $C(X)$ consisting of bounded functions, functions vanishing at infinity, functions with compact support, and bounded functions with bounded support, respectively. Let $\operatorname{Lip}(X)$ and $\operatorname{Lip}_{b}(X)$ denote the set of Lipschitz continuous functions, and the set of bounded Lipschitz continuous functions, respectively. For $f \in \operatorname{Lip}(X)$, we denote by $\operatorname{Lip}_{X}(f)$ the global Lipschitz constant of $f$. The set $\mathcal{P}(X)$ denotes all Borel probability measures on $X$. The set of continuous functions on $[0, \infty)$ valued in $X$ is denoted by $C([0, \infty), X)$.

A continuous curve $\gamma:[a, b] \rightarrow X$ is connecting $x$ and $y$ if $\gamma_{a}=x$ and $\gamma_{b}=y$. A continuous curve $\gamma:[a, b] \rightarrow X$ is a minimal geodesic if

$$
d\left(\gamma_{t}, \gamma_{s}\right)=\frac{|s-t|}{|b-a|} d\left(\gamma_{a}, \gamma_{b}\right) \quad a \leq t \leq s \leq b .
$$

In particular, if $\frac{d\left(\gamma_{a}, \gamma_{b}\right)}{|b-a|}$ can be replaced by 1 , we say that $\gamma$ is unit-speed. A metric space $X$ is called geodesic if for any two points $x, y \in X$, there exists a minimal geodesic $\left\{\gamma_{t}\right\}_{t \in[0,1]}$ connecting $x$ and $y$.

Let $\operatorname{supp}[m]=\left\{x \in X: m\left(B_{r}(x)\right)>0, \forall r>0\right\}$ denote the support of $m$. Let $\left(Y, d_{Y}\right)$ be a complete separable metric space. For a Borel measurable map $f: X \rightarrow Y$, let $f_{\#} m$ denote the push-forward measure on $Y: f_{\#} m(B)=m\left(f^{-1}(B)\right)$ for any Borel set $B \in \mathscr{B}(Y)$.

\section{2 $\quad L^{p}$-Wasserstein space}

Let $\left(X_{i}, d_{i}\right)(i=1,2)$ be complete separable metric spaces and $1 \leq p<\infty$. For $\mu_{i} \in \mathcal{P}\left(X_{i}\right)$, a probability measure $q \in \mathcal{P}\left(X_{1} \times X_{2}\right)$ is called a coupling of $\mu_{1}$ and $\mu_{2}$ if $\pi_{1 \#} q=\mu_{1}$ and $\pi_{2 \#} q=\mu_{2}$, whereby $\pi_{i}(i=1,2)$ is the projection $\pi_{i}: X_{1} \times X_{2} \rightarrow X_{i}$ as $\left(x_{1}, x_{2}\right) \mapsto x_{i}$. We denote by $\Pi(\mu, \nu)$ the set of all coupling of $\mu$ and $\nu$.

Let $(X, d)$ be a complete separable metric space. Let $\mathcal{P}_{p}(X)$ be the subset of $\mathcal{P}(X)$ consisting of all Borel probability measures $\mu$ on $X$ with finite $p$-th moment:

$$
\int_{X} d^{p}(x, \bar{x}) d \mu(x)<\infty \text { for some (and thus any) } \bar{x} \in X .
$$

We equip $\mathcal{P}_{p}(X)$ with the transportation distance $W_{p}$, called $L^{p}$-Wasserstein distance, defined as follows:

$$
W_{p}(\mu, \nu)=\left(\inf _{q \in \Pi(\mu, \nu)} \int_{X \times X} d^{p}(x, y) d q(x, y)\right)^{1 / p} .
$$

A coupling $q \in \Pi(\mu, \nu)$ is called an optimal coupling if $q$ attains the infimum in the equality (2.1). It is known that, for any $\mu, \nu$, there always exists an optimal coupling 
$q$ of $\mu$ and $\nu$ (e.g., [70, §4]). It is also known that $\left(\mathcal{P}_{p}(X), W_{p}\right)$ is a complete separable geodesic metric space for $1<p<\infty$ if $(X, d)$ is a complete separable geodesic metric space (e.g., [70, Theorem 6.18]).

\subsection{Pointed measured Gromov convergence}

In this subsection, we recall the definition of pmG convergence introduced in GigliMondino-Savaré [30].

Definition 2.1 (pmG Convergence [30]). A sequence of p.m.m. spaces $\left\{\mathcal{X}_{n}=\right.$ $\left.\left(X_{n}, d_{n}, m_{n}, \bar{x}_{n}\right)\right\}_{n \in \mathbb{N}}$ satisfying (1.1) is convergent to $\mathcal{X}_{\infty}=\left(X_{\infty}, d_{\infty}, m_{\infty}, \bar{x}_{\infty}\right)$ in the pointed measured Gromov (pmG) sense if there exist a complete separable metric space $(X, d)$ and isometric embeddings $\iota_{n}: X_{n} \rightarrow X(n \in \overline{\mathbb{N}}:=\mathbb{N} \cup\{\infty\})$ satisfying

$$
\iota_{n}\left(\bar{x}_{n}\right) \rightarrow \iota_{\infty}\left(\bar{x}_{\infty}\right) \in \operatorname{supp}\left[m_{\infty}\right], \quad \text { and } \int_{X} f d\left(\iota_{n \#} m_{n}\right) \rightarrow \int_{X} f d\left(\iota_{\infty} m_{\infty}\right),
$$

for any bounded continuous function $f: X \rightarrow \mathbb{R}$ with bounded support.

Remark 2.2. We would like to remark on the pmG convergence in Definition 2.1.

(i) In general, the pmG convergence is strictly weaker than the pointed measured Gromov-Hausdorff (pmGH) convergence ([30, Theorem 3.30, Example 3.31]). However, if $\operatorname{supp}\left[m_{\infty}\right]=X_{\infty}$ and $\left\{\mathcal{X}_{n}\right\}_{n \in \mathbb{N}}$ satisfies a uniform doubling condition, then the two notions of pmG and pmGH coincide (see [30, Theorem 3.33]).

(ii) The pmG convergence is metrizable by the distance $p \mathbb{G}_{W}$ on the collection $\mathbb{X}$ of all isomorphism classes of p.m.m. spaces (see [30, Definition 3.13]). The space $\left(\mathbb{X}, p \mathbb{G}_{W}\right)$, moreover, is a complete and separable metric space (see [30, Theorem 3.17]).

\subsection{RCD spaces}

In this subsection, we recall the definition of the $\operatorname{RCD}(K, \infty)$ condition. We also recall several properties satisfied on $\operatorname{RCD}(K, \infty)$ spaces. See [2] for more comprehensive accounts of this field.

\subsubsection{Relative entropy}

In this subsection, we recall the definition of the relative entropy functional Ent ${ }_{m}$ : $\mathcal{P}_{2}(X) \rightarrow \overline{\mathbb{R}}:=\mathbb{R} \cup\{+\infty\}:$

$$
\operatorname{Ent}_{m}(\mu)= \begin{cases}\int_{X} \frac{d \mu}{d m} \log \left(\frac{d \mu}{d m}\right) d m, & \text { if } \mu \ll m \\ +\infty, & \text { otherwise }\end{cases}
$$

Here $d \mu / d m$ denotes the Radon-Nikodym derivative. Let us write $D\left(\operatorname{Ent}_{m}\right):=\{\mu \in$ $\left.\mathcal{P}_{2}(X): \operatorname{Ent}_{m}(\mu)<\infty\right\}$. Although $m$ might not be a probability measure, the entropy $\mathrm{Ent}_{m}$ is well-defined and lower-semicontinuous thanks to condition (1.2). Indeed, by recalling (1.3):

$$
z:=\int_{X} e^{-C d^{2}(x, \bar{x})} d m(x), \quad \text { so that } \quad \widetilde{m}:=\frac{1}{z} e^{-C d^{2}(\cdot, \bar{x})} m,
$$

we can check that, for any $\rho m=\mu \in D\left(\operatorname{Ent}_{m}\right)$ with $\rho=\frac{d \mu}{d m}$, it holds that $\mu=z \rho e^{\mathrm{C} d^{2}(\cdot, \bar{x})} \widetilde{m}$. Therefore we obtain

$$
\operatorname{Ent}_{m}(\mu)=\operatorname{Ent}_{\widetilde{m}}(\mu)-\mathrm{C} \int_{X} d^{2}(\cdot, \bar{x}) d \mu-\log z,
$$


which implies that Ent ${ }_{m}$ is well-defined and lower-semicontinuous with respect to $W_{2}$ topology.

\subsubsection{Cheeger energy}

In this subsection, we recall the Cheeger energy $\mathrm{Ch}$ on $(X, d, m, \bar{x})$. For $f \in \operatorname{Lip}(X)$, the local Lipschitz constant $|\nabla f|: X \rightarrow \mathbb{R}$ is defined as follows:

$$
|\nabla f|(x)= \begin{cases}\limsup _{y \rightarrow x} \frac{|f(y)-f(x)|}{d(y, x)}, & \text { if } x \text { is not isolated, } \\ 0, & \text { otherwise. }\end{cases}
$$

Then we now recall the definition of Cheeger energy: (see [29, 3, 5])

$$
\begin{aligned}
\operatorname{Ch}(f) & =\frac{1}{2} \inf \left\{\liminf _{n \rightarrow \infty} \int\left|\nabla f_{n}\right|^{2} d m: f_{n} \in \operatorname{Lip}(X) \cap L^{2}(X, m), \int_{X}\left|f_{n}-f\right|^{2} d m \rightarrow 0\right\} \\
W^{1,2}(X, d, m) & =\left\{f \in L^{2}(X, m): \operatorname{Ch}(f)<\infty\right\} .
\end{aligned}
$$

If $\mathrm{Ch}(f)<\infty$, then the Cheeger energy can be written as an integral form by minimal weak upper gradient $|\nabla f|_{w}$ (see [5, 3]):

$$
\operatorname{Ch}(f)=\frac{1}{2} \int_{X}|\nabla f|_{w}^{2} d m, \quad \forall f \in W^{1,2}(X, d, m) .
$$

\subsection{3 $\mathrm{RCD}(K, \infty)$ spaces}

In this subsection, we recall the $\mathrm{CD}(K, \infty) / \mathrm{RCD}(K, \infty)$ condition.

Definition 2.3. The $\mathrm{CD}(K, \infty) / \mathrm{RCD}(K, \infty)$ conditions are defined as follows:

(i) $(\mathrm{CD}(K, \infty))$ [[65], [47]]

We say that $(X, d, m)$ satisfies the curvature-dimension condition $\operatorname{CD}(K, \infty)$ for $K \in$ $\mathbb{R}$ if, for each $\mu_{0}, \mu_{1} \in D\left(\right.$ Ent $\left._{m}\right)$, there exists a $W_{2}$-geodesic $\left\{\mu_{t}\right\}_{t \in[0,1]} \subset D\left(\right.$ Ent $\left._{m}\right)$ connecting $\mu_{0}$ and $\mu_{1}$ so that

$$
\operatorname{Ent}_{m}\left(\mu_{t}\right) \leq(1-t) \operatorname{Ent}_{m}\left(\mu_{0}\right)+t \operatorname{Ent}_{m}\left(\mu_{1}\right)-\frac{K}{2} t(1-t) W_{2}^{2}\left(\mu_{0}, \mu_{1}\right) .
$$

(ii) $(\operatorname{RCD}(K, \infty))[[29$, Remark 4.20], [6, Theorem 5.1], [3, Theorem 6.1]]

We say that $(X, d, m)$ satisfies the Riemannian curvature-dimension condition $\operatorname{RCD}(K, \infty)$ if the following two conditions hold:

(ii-a) $\mathrm{CD}(K, \infty)$

(ii-b) the infinitesimal Hilbertianity, that is, the Cheeger energy $\mathrm{Ch}$ is a quadratic form:

$$
2 \mathrm{Ch}(u)+2 \mathrm{Ch}(v)=\operatorname{Ch}(u+v)+\operatorname{Ch}(u-v),
$$

for any $u, v \in W^{1,2}(X, d, m)$.

It is known that $\mathrm{CD}(K, \infty) / \mathrm{RCD}(K, \infty)$ conditions are stable under the pmG convergence.

Theorem 2.4 (Stability of the $\operatorname{RCD}(K, \infty)$ condition $[65,6,3]$ ). Let $\left\{\mathcal{X}_{n}\right\}_{n \in \mathbb{N}}$ be a sequence of $\operatorname{RCD}(K, \infty)$ spaces. If $\mathcal{X}_{n}$ converges to $\mathcal{X}_{\infty}$ in the pmG sense, then the limit space $\mathcal{X}_{\infty}$ is also an $\operatorname{RCD}(K, \infty)$ space. 


\subsection{4 $W_{2}$-gradient flow of relative entropy}

In this subsection, following $[3,5]$, we recall the heat flow on the $L^{2}$-Wasserstein space $\left(\mathcal{P}_{2}(X), W_{2}\right)$, which is constructed by the gradient flow of the relative entropy functional. We also recall the stability of the heat flows under the pmG convergence.

The descendent slope $\mid D^{-}$Ent $_{m} \mid: \mathcal{P}_{2}(X) \rightarrow[-\infty, \infty]$ of the relative entropy Ent $_{m}$ is defined as follows:

$$
\left|D^{-} \operatorname{Ent}_{m}\right|(\mu)= \begin{cases}\limsup _{W_{2}(\nu, \mu) \rightarrow 0} \frac{\left(\operatorname{Ent}_{m}(\mu)-\operatorname{Ent}_{n}(\nu)\right)^{+}}{W_{2}(\nu, \mu)}, & \text { if } \mu \in D\left(\operatorname{Ent}_{m}\right), \\ 0, & \text { if } \mu \text { is isolated in } \mathcal{P}_{2}(X), \\ +\infty, & \text { if } \mu \in \mathcal{P}_{2}(X) \backslash D\left(\operatorname{Ent}_{m}\right) .\end{cases}
$$

Here $(\cdot)^{+}$denotes the positive part. Let $\mathcal{X}=(X, d, m, \bar{x})$ be a $\operatorname{CD}(K, \infty)$ space and $\bar{\mu} \in D\left(\right.$ Ent $\left._{m}\right)$. A curve $\mu:[0, \infty) \rightarrow D\left(\right.$ Ent $\left._{m}\right) \subset \mathcal{P}_{2}(X)$ is said to be the $W_{2}$-gradient flow of Ent $_{m}$ starting at $\bar{\mu}$ if $\mu$ is locally absolutely continuous in $\left(\mathcal{P}_{2}(X), W_{2}\right)$ with $\mu_{0}=\bar{\mu}$ and

$$
\operatorname{Ent}_{\mathrm{m}}\left(\mu_{t}\right)=\operatorname{Ent}_{\mathrm{m}}\left(\mu_{s}\right)+\frac{1}{2} \int_{t}^{s}\left|\dot{\mu}_{r}\right|^{2} d r+\frac{1}{2} \int_{t}^{s}\left|D^{-} \operatorname{Ent}_{m}\right|^{2}\left(\mu_{r}\right) d r, \quad 0<\forall t<\forall s .
$$

Under the $\operatorname{CD}(K, \infty)$ condition, it is known that the gradient flow $\mu_{t}=\mathcal{H}_{t} \bar{\mu}$ of the relative entropy exists uniquely for any initial measure $\bar{\mu} \in \overline{D\left(\text { Ent }_{m}\right)}$ and for any $t \geq 0([3,5])$. Here $\overline{D\left(\text { Ent }_{m}\right)}$ means the closure of $D\left(\right.$ Ent $\left._{m}\right)$. We call $\left\{\mathcal{H}_{t}\right\}_{t \geq 0}$ heat flow on $\mathcal{P}_{2}(X)$.

Theorem 2.5 (Theorem 7.7 in [30] (Stability of heat flows)). Let $\left\{\mathcal{X}_{n}=\left(X_{n}, d_{n}, m_{n}, \bar{x}_{n}\right)\right\}_{n \in \mathbb{N}}$ be a sequence of $\operatorname{RCD}(K, \infty)$ spaces converging to $\mathcal{X}_{\infty}=\left(X_{\infty}, d_{\infty}, m_{\infty}, \bar{x}_{\infty}\right)$ in the pmG sense. If $\mu_{n} \in \mathcal{P}_{2}\left(\operatorname{supp}\left[m_{n}\right]\right) \subset \mathcal{P}_{2}(X)$ converges to $\mu_{\infty} \in \mathcal{P}_{2}\left(\operatorname{supp}\left[m_{\infty}\right]\right) \subset \mathcal{P}_{2}(X)$ in the $W_{2}$-sense:

$$
W_{2}\left(\iota_{n \#} \mu_{n}, \iota_{\infty \#} \mu_{\infty}\right) \rightarrow 0, \quad n \rightarrow \infty,
$$

then the solution $\mu_{t}^{n}=\mathcal{H}_{t}^{n}\left(\mu_{n}\right)$ of the heat flow starting at $\mu_{n}$ converges to the limit one $\mu_{t}^{\infty}=\mathcal{H}_{t}^{\infty}\left(\mu_{\infty}\right)$ in the $W_{2}$-sense:

$$
W_{2}\left(\iota_{n \#} \mu_{t}^{n}, \iota_{\infty \#} \mu_{t}^{\infty}\right) \rightarrow 0, \quad n \rightarrow \infty, \quad \forall t \geq 0 .
$$

Here $\iota_{n}$ is an embedding $X_{n} \rightarrow X$ corresponding to the pmG convergence (see Definition 2.1).

\subsection{5 $L^{2}$-gradient flow of Cheeger energy}

We now recall the $L^{2}$-gradient flow of Cheeger energy by Hilbertian theory of gradient flows (see e.g., [7]). We also recall the important fact that the heat flow in the previous section and the $L^{2}$-gradient flow of Cheeger energy in this section coincide under the $\mathrm{CD}(K, \infty)$ condition.

For $f_{0} \in L^{2}(X ; m)$, there exists a locally Lipschitz map $t \mapsto f_{t}=H_{t} f_{0} \in L^{2}(X ; m)$ with $f_{t} \rightarrow f_{0}$ as $t \downarrow 0$ whose derivative satisfies

$$
\frac{d}{d t} f_{t} \in-\partial^{-} \mathrm{Ch}\left(f_{t}\right), \quad \text { a.e. }-t>0 \text {. }
$$

Here the subdifferential $\partial^{-}$Ch of convex analysis is the multi-valued operator in $L^{2}(X ; m)$ defined at all elements of the domain of the Cheeger energy $f \in W^{1,2}(X, d, m)$ by the family of inequalities

$$
h \in \partial^{-} \mathrm{Ch}(f) \Longleftrightarrow \int_{X} h(g-f) d m \leq \mathrm{Ch}(g)-\mathrm{Ch}(f), \quad \forall g \in L^{2}(X ; m) .
$$


The map $H_{t}: f_{0} \mapsto f_{t}$ is uniquely determined by (2.4) and define a contraction semigroup (not necessarily linear) on $L^{2}(X ; m)$. The flow $f_{0} \mapsto f_{t}=H_{t} f$ is called $L^{2}$-gradient flow of the Cheeger energy, and the semigroup $\left\{H_{t}\right\}_{t \geq 0}$ is called heat semigroup.

We recall that the $L^{2}$-gradient flows of Cheeger energies and the $W_{2}$-gradient flow of entropies are equivalent under the $\operatorname{CD}(K, \infty)$ condition.

Theorem 2.6 ([5, Theorem 9.3] (see also [3])). Let $\mathcal{X}=(X, d, m, \bar{x})$ be a p.m.m. space satisfying the $\mathrm{CD}(K, \infty)$ condition. If $\mu_{0}=f_{0} m \in \mathcal{P}_{2}(X)$ with $f_{0} \in L^{2}(X ; m)$, then

$$
\mathcal{H}_{t}\left(\mu_{0}\right)=\left(H_{t} f_{0}\right) m, \quad \forall t \geq 0 .
$$

\subsection{6 $\mathrm{RCD}^{*}(K, N)$ spaces}

In this subsection, we recall the definition of the $\operatorname{RCD}^{*}(K, N)$ condition and several properties satisfied by $\operatorname{RCD}^{*}(K, N)$ spaces (see $[29,8,22]$ for more details).

For each $\theta \in[0, \infty)$, we define the following functions

$$
\Theta_{\kappa}(\theta)= \begin{cases}\frac{\sin (\sqrt{\kappa} \theta)}{\sqrt{\kappa}}, & \text { if } \quad \kappa>0, \\ \theta, & \text { if } \quad \kappa=0, \\ \frac{\sinh (\sqrt{-\kappa} \theta)}{\sqrt{-\kappa}}, & \text { if } \quad \kappa<0 .\end{cases}
$$

We define the following functions: for $t \in[0,1]$,

$$
\sigma_{\kappa}^{(t)}(\theta)= \begin{cases}\frac{\Theta_{\kappa}(t \theta)}{\Theta_{\kappa}(\theta)}, & \text { if } \quad \kappa \theta^{2} \neq 0 \text { and } \kappa \theta^{2}<\pi^{2}, \\ t, & \text { if } \kappa \theta^{2}=0, \\ +\infty, & \text { if } \kappa \theta^{2} \geq \pi^{2} .\end{cases}
$$

Let $P_{\infty}(X, d, m)$ be the subset of $\mathcal{P}_{2}(X)$ consisting of $\mu$ which is absolutely continuous with respect to $m$ and has bounded support.

Definition $2.7\left(\mathrm{CD}^{*}(K, N)\right.$ and $\left.\mathrm{RCD}^{*}(K, N)[11,29]\right)$.

(i) A metric measure space $(X, d, m)$ is said to satisfy the reduced curvature-dimension condition $\mathrm{CD}^{*}(K, N)$ for $K, N \in \mathbb{R}$ with $1<N<\infty$ if, for each pair $\mu_{0}=\rho_{0} m$ and $\mu_{1}=\rho_{1} m$ in $P_{\infty}(X, d, m)$, there exist an optimal coupling $q$ of $\mu_{0}$ and $\mu_{1}$ and a geodesic $\mu_{t}=\rho_{t} m(t \in[0,1])$ in $\left(P_{\infty}(X, d, m), W_{2}\right)$ connecting $\mu_{0}$ and $\mu_{1}$ so that, for all $t \in[0,1]$ and $N^{\prime} \geq N$, we have

$$
\int \rho_{t}^{-\frac{1}{N^{\prime}}} d \mu_{t} \geq \int_{X \times X}\left[\sigma_{\frac{K}{N^{\prime}}}^{(1-t)}\left(d\left(x_{0}, x_{1}\right)\right) \rho_{0}^{-\frac{1}{N^{\prime}}}\left(x_{0}\right)+\sigma_{\frac{K}{N^{\prime}}}^{(t)}\left(d\left(x_{0}, x_{1}\right)\right) \rho_{1}^{-\frac{1}{N^{\prime}}}\left(x_{1}\right)\right] d q\left(x_{0}, x_{1}\right) .
$$

(ii) A metric measure space $(X, d, m)$ is said to satisfy the Riemannian curvaturedimension condition $\operatorname{RCD}^{*}(K, N)$ if the following two conditions hold:

(ii-a) $\mathrm{CD}^{*}(K, N)$

(ii-b) the infinitesimal Hilbertianity, that is the Cheeger energy $\mathrm{Ch}$ is a quadratic form:

$$
\begin{array}{r}
2 \operatorname{Ch}(u)+2 \operatorname{Ch}(v)=\operatorname{Ch}(u+v)+\operatorname{Ch}(u-v), \\
\forall u, v \in W^{1,2}(X, d, m) .
\end{array}
$$

Remark 2.8. The $\operatorname{RCD}^{*}(K, N)$ condition is stronger than the $\operatorname{RCD}(K, \infty)$ condition. If $X$ is an $\operatorname{RCD}^{*}(K, N)$ space, then $X$ is locally compact by the local volume doubling property according to Bishop-Gromov inequality [22, Proposition 3.6] (see also [66, Corollary 2.4]). 
The $\operatorname{RCD}^{*}(K, N)$ condition is stable under the pmG convergence.

Theorem 2.9 (Stability of $\operatorname{RCD}^{*}(K, N)$ [29]). Let $\left\{\mathcal{X}_{n}\right\}_{n \in \mathbb{N}}$ be a sequence of $\operatorname{RCD}^{*}(K, N)$ spaces. If $\mathcal{X}_{n}$ converges to $\mathcal{X}_{\infty}$ in the pmG sense, then $\mathcal{X}_{\infty}$ is also an $\operatorname{RCD}^{*}(K, N)$ space.

\section{5 $\quad L^{2}$-convergence of heat semigroups under the PmG convergence}

In Gigli-Mondino-Savaré [30], they introduced $L^{2}$-convergences on varying metric measure spaces and showed a convergence of heat semigroups in this sense under the pmG convergence of the underlying spaces with the $\operatorname{RCD}(K, \infty)$ condition. We recall their results briefly.

Definition 2.10 (See [30, Definition 6.1]). Let $\left\{\left(X_{n}, d_{n}, m_{n}, \bar{x}_{n}\right)\right\}_{n \in \mathbb{N}}$ be a sequence of p.m.m. spaces. Assume that $\left(X_{n}, d_{n}, m_{n}, \bar{x}_{n}\right)$ converges to $\left(X_{\infty}, d_{\infty}, m_{\infty}, \bar{x}_{\infty}\right)$ in the pmG sense. Let $(X, d)$ be a complete separable metric space and $\iota_{n}: \operatorname{supp}\left[m_{n}\right] \rightarrow X$ be isometries as in Definition 2.1. We identify $\left(X_{n}, d_{n}, m_{n}\right)$ with $\left(\iota_{n}\left(X_{n}\right), d, \iota_{n \#} m_{n}\right)$ and omit $\iota_{n}$.

(i) We say that $u_{n} \in L^{2}\left(X, m_{n}\right)$ converges weakly to $u_{\infty} \in L^{2}\left(X, m_{\infty}\right)$ if the following hold:

$$
\sup _{n \in \mathbb{N}} \int\left|u_{n}\right|^{2} d m_{n}<\infty \quad \text { and } \int \phi u_{n} d m_{n} \rightarrow \int \phi u_{\infty} d m_{\infty} \quad \forall \phi \in C_{b s}(X),
$$

whereby recall that $C_{b s}(X)$ denotes the set of bounded continuous functions with bounded support.

(ii) We say that $u_{n} \in L^{2}\left(X, m_{n}\right)$ converges strongly to $u_{\infty} \in L^{2}\left(X, m_{\infty}\right)$ if $u_{n}$ converges weakly to $u_{\infty}$ and the following holds:

$$
\limsup _{n \rightarrow \infty} \int\left|u_{n}\right|^{2} d m_{n} \leq \int\left|u_{\infty}\right|^{2} d m_{\infty}
$$

Let $\left\{H_{t}^{n}\right\}_{t \geq 0}$ be the $L^{2}\left(X, m_{n}\right)$-semigroup corresponding to the Cheeger energy $\mathrm{Ch}_{n}$. Then the following theorem states that $\left\{H_{t}^{n}\right\}_{t \geq 0}$ convergence strongly in $L^{2}$ under the pmG convergence of the underlying spaces.

Theorem 2.11 (See [30, Theorem 6.11]). Let $\left\{\left(X_{n}, d_{n}, m_{n}, \bar{x}_{n}\right)\right\}_{n \in \mathbb{N}}$ be a sequence of p.m.m. spaces satisfying the $\operatorname{RCD}(K, \infty)$ for all $n \in \mathbb{N}$. Then, for any $u_{n} \in L^{2}\left(X, m_{n}\right)$ converging strongly to $u_{\infty} \in L^{2}\left(X, m_{\infty}\right)$, we have, for any $t>0$

$$
H_{t}^{n} u_{n} \text { converges strongly to } H_{t}^{\infty} u_{\infty} \text { in the sense of Definition 2.10. }
$$

Note that, in [30, Theorem 6.11], the above Theorem 2.11 was stated without the condition of the infinitesimal Hilbertian.

\section{Brownian motion on RCD spaces}

\subsection{Brownian motions on $\operatorname{RCD}(K, \infty)$ spaces}

Let $(X, d, m)$ satisfy the $\operatorname{RCD}(K, \infty)$ condition. Let $\delta_{x}$ denote the unit mass at $x \in X$, and define a kernel $p(t, x, d y)$ by the action of the heat flow (see Subsection 2.4.4)

$$
p(t, x, d y):=\mathcal{H}_{t}\left(\delta_{x}\right) \quad \forall t>0, x \in X .
$$

Then we have that (see $[6,3])$

$$
p(t, x, d y) \text { is absolutely continuous with respect to } m \text { for any } t>0,
$$


and we denote the density by $p(t, x, y)$. By [6, Theorem 6.1] and [3] (for the case of $\sigma$-finite reference measures), the density $p(t, x, y)$ is symmetric in $x$ and $y$, and satisfies the Chapman-Kolmogorov formula. Moreover, the following action of semigroup $\left\{P_{t}\right\}_{t \geq 0}$

$$
P_{t} f(x):=\int_{X} f(y) d \mathcal{H}_{t}\left(\delta_{x}\right)(d y)
$$

is a version of the linear heat semigroup $\left\{H_{t}\right\}_{t \geq 0}$ defined as the gradient flow of the Cheeger energy $C h$ (see Subsection 2.4.5) for any $f \in L^{2}(X ; m)$. Furthermore $P_{t}$ is an extension of $H_{t}$ to a continuous contraction semigroup in $L^{1}(X ; m)$ which is point-wise everywhere defined on $\operatorname{supp}[m]$ if $f \in L^{\infty}(X ; m)$ since $P_{t} f$ becomes Lipschitz continuous on $\operatorname{supp}[m]$ whenever $f \in L^{\infty}(X ; m)$ (see [6, Theorem 6.5] and [3, Theorem 7.3]). We call $p(t, x, d y)$ and $p(t, x, y)$ the heat kernel and the heat kernel density, respectively. By the Kolmogorov extension theorem, we can construct a family of probability measures $\left\{\mathbb{P}^{x}\right\}_{x \in X}$ on $X^{[0, \infty)}$ and a system of Markov processes $\left(\left\{\mathbb{P}^{x}\right\}_{x \in X},\left\{B_{t}\right\}_{t \geq 0}\right)$ on $X$ with respect to $p(t, x, d y)$.

On the other hand, we can define a Dirichlet form (i.e., a symmetric closed Markovian bilinear form) $(\mathcal{E}, \mathcal{F})$ induced by the Cheeger energy $\mathrm{Ch}$ as follows:

$$
\mathcal{E}(u, v)=\frac{1}{4}(\operatorname{Ch}(u+v)-\operatorname{Ch}(u-v)), \quad u, v \in \mathcal{F}=W^{1,2}(X, d, m) .
$$

By [6, Lemma 6.7] (see [3, Theorem 7.2] for $\sigma$-finite reference measures), the form $(\mathcal{E}, \mathcal{F})$ becomes a quasi-regular conservative strongly-local symmetric Dirichlet form below. See [6, Proposition 4.11] for the strong locality, and the conservativeness follows from the volume growth estimate (1.2) and Sturm's conservativeness test [62, Theorem 4].

Therefore, by [49, Theorem IV 3.5, V1.5], there exists a family of probability measures $\left\{\mathbb{Q}^{x}\right\}_{x \in X}$ on $C([0, \infty) ; X)$ and a system $\left(\left\{\mathbb{Q}^{x}\right\}_{x \in X},\left\{B_{t}^{\prime}\right\}_{t \geq 0}\right)$ of conservative diffusion processes so that

$$
\mathbb{E}_{\mathrm{Q}}^{x}\left(f\left(B_{t}^{\prime}\right)\right)=P_{t} f, \quad \forall f \in L^{2}(X ; m) \cap \mathcal{B}_{b}(X), \quad \forall t \geq 0, \quad \forall x \in X \backslash \mathcal{N} .
$$

Here $\mathbb{E}_{\mathbb{Q}}^{x}$ denotes the expectation with respect to $\mathbb{Q}^{x}$ and $\mathcal{N}$ is a set of zero-capacity with respect to $(\mathcal{E}, \mathcal{F})$. By conservative diffusion process, we mean that $\left(\mathbb{Q}^{x},\left\{B_{t}^{\prime}\right\}_{t \geq 0}\right)$ is a strong Markov process whose sample path is continuous, which means $B^{\prime} \in C([0, \infty) ; X)$ $\mathbb{Q}^{x}$-almost surely for every $x \in X$. The systems of Markov processes corresponding to $(\mathcal{E}, \mathcal{F})$ are unique up to zero-capacity sets with respect to starting points $x$. Namely, if there is another system of diffusion processes $\left(\left\{\mathbb{R}^{x}\right\}_{x \in X},\left\{S_{t}\right\}_{t \geq 0}\right)$ corresponding to $(\mathcal{E}, \mathcal{F})$, then the laws of $\left(\mathbb{Q}^{x},\left\{B_{t}^{\prime}\right\}_{t \geq 0}\right)$ and $\left(\mathbb{R}^{x},\left\{S_{t}\right\}_{t \geq 0}\right)$ coincide for every $x \in X \backslash \mathcal{N}$ with some set $\mathcal{N}$ of zero-capacity. Note that, if $\left\{P_{t}\right\}_{t \geq 0}$ is a Feller semigroup, then $\mathcal{N}$ can be taken as an empty set $\emptyset$, and the diffusion process can be defined uniquely with respect to every starting point $x$. See, e.g., [27, Chapter 7, A.2] and [49, Chapter IV] for more comprehensive accounts.

Let $\mathbb{P}_{*}^{x}$ denote the outer measure of $\mathbb{P}^{x}$ on all subsets of $X^{[0, \infty)}$. By the same argument of [9, Proof of (c) in Theorem 1.2], we have that

$$
\mathbb{P}_{*}^{x}(C((0, \infty) ; X))=1 \quad \text { for every } x \in X \text { (not only quasi-every } x \in X \text { ). }
$$

This property is due to the absolute continuity of the heat kernel $p(t, x, d y)$ with respect to $m$ for any $t>0$. Note that, by the conservativeness and the strong locality of the Dirichlet form $(\mathcal{E}, \mathcal{F})$, we know that $\mathbb{Q}^{x}(C([0, \infty) ; X))=1$ for quasi-every $x \in X$ (this holds also for $\left.\mathbb{P}_{*}^{x}\right)$. We write simply $\mathbb{P}^{x}$ for $\mathbb{P}_{*}^{x}$. The systems of Markov processes $\left(\left\{\mathbb{P}^{x}\right\}_{x \in X},\left\{B_{t}\right\}_{t \geq 0}\right)$ and $\left(\left\{\mathbb{Q}^{x}\right\}_{x \in X},\left\{B_{t}^{\prime}\right\}_{t \geq 0}\right)$ coincide except on zero-capacity sets. In this paper, we adopt $\left(\left\{\mathbb{P}^{x}\right\}_{x \in X},\left\{B_{t}\right\}_{t \geq 0}\right)$ for representing a system of Brownian motions. 
Remark 3.1. The diffusion process defined above is conventionally called Brownian motion ([6]), but this may indicate other diffusion processes than the standard Brownian motion in some situations. For instance, when we take $(X, d, m)=\left(\mathbb{R}^{d},\|\cdot\|_{2}\right.$, $\left.\frac{1}{(2 \pi)^{d / 2}} \exp \left\{-\frac{1}{2}\|x\|_{2}^{2}\right\} d x\right)$ whereby $d x$ denotes the Lebesgue measure, and $\|\cdot\|_{2}$ denotes the Euclidean distance. Then $(X, d, m)$ satisfies $\operatorname{RCD}(0, \infty)$ and the diffusion induced by the Cheeger energy coincides with what is known as the Ornstein-Uhlenbeck process, which is different from the standard Brownian motion on $\mathbb{R}^{d}$.

\subsection{Brownian motions on $\operatorname{RCD}^{*}(K, N)$ spaces}

In this subsection, we show the Feller property of the heat semigroup on $\operatorname{RCD}^{*}(K, N)$ spaces.

Proposition 3.2. Under the $\operatorname{RCD}^{*}(K, N)$ condition, the heat semigroup $\left\{H_{t}\right\}_{t \geq 0}$ has a Feller modification. That is, there exists a semigroup $\left\{P_{t}\right\}_{t \geq 0}$ so that $P_{t} f=H_{t} f m$-a.e. for any $f \in L^{2}(X, m)$ and any $t>0$ and the following conditions hold:

(F-1) For any $f \in C_{\infty}(X), P_{t} f \in C_{\infty}(X)$ for any $t>0$.

(F-2) For any $f \in C_{\infty}(X),\left\|P_{t} f-f\right\|_{\infty} \rightarrow 0 \quad t \downarrow 0$.

Remark 3.3. The following proof is the result of a private communication with Prof. Kazuhiro Kuwae. Although the proof might be already known in some literature, we could not find good references and we give the proof for the sake of reader's convenience.

Proof. By [6, (iii) in Theorem 6.1], there exists a semigroup $\left\{P_{t}\right\}_{t \geq 0}$ which is a modification of $\left\{H_{t}\right\}_{t \geq 0}$ so that $P_{t} f \in \operatorname{Lip}_{b}(X)$ if $f \in L^{\infty}(X, m)$. Before checking (F-1) and (F-2), we first give a heat kernel estimate. By [35, Theorem 1.2], we have the following Gaussian heat kernel estimate: there exist positive constants $C_{i}=C_{i}(N, K)$ for $i=1,2,3$ depending only on $N, K$ so that

$$
p(t, x, y) \leq \frac{C_{1}}{m\left(B_{\sqrt{t}}(y)\right)} \exp \left\{-C_{2} \frac{d(x, y)^{2}}{t}-C_{3} t\right\},
$$

for all $x, y \in X$ and $0<t$. Here the heat kernel $p(t, x, y)$ means the integral kernel of the heat semigroup $P_{t} f(x)=\int_{X} f p(t, x, y) m(d y)$ for $t>0$.

We now show condition (F-1). We already know $P_{t} f \in C_{b}(X)$, so it suffices to show that $P_{t} f$ vanishes at infinity for $f \in C_{\infty}(X)$ and $t>0$, which is an easy consequence of (3.3) as follows: we may assume that $f$ is compactly supported since every element in $C_{\infty}(X)$ can be approximated by elements in $C_{0}(X)$ with respect to the uniform norm. Let $K \supset \operatorname{supp}[f]$ be a compact set. By (3.3) and $\inf _{y \in K} m\left(B_{\sqrt{t}}(y)\right)>0$ (by the lower semi-continuity of $m\left(B_{r}(x)\right)$ in $\left.x\right)$, we see that, for any $\varepsilon>0$, there exists a compact set $K^{\prime} \subset X$ so that

$$
\begin{aligned}
\left|P_{t} f(x)\right| & \leq \int_{K} p(t, x, y)|f(y)| m(d y) \\
& <\|f\|_{\infty} \int_{K} \frac{C_{1}}{m\left(B_{\sqrt{t}}(y)\right)} \exp \left\{-C_{2} \frac{d(x, y)^{2}}{t}-C_{3} t\right\} m(d y) \\
& <\frac{C_{1}\|f\|_{\infty}}{\inf _{y \in K} m\left(B_{\sqrt{t}}(y)\right)} \int_{K} \exp \left\{-C_{2} \frac{d(x, y)^{2}}{t}-C_{3} t\right\} m(d y) \\
& \leq \varepsilon \quad\left(\forall x \in X \backslash K^{\prime}\right) .
\end{aligned}
$$

Thus we have proved (F-1).

Now we prove (F-2). We may assume $f \in C_{0}(X)$. Let $K \supset \operatorname{supp}[f]$ be a compact set. For given $\varepsilon>0$, take $\delta>0$ so that $|f(x)-f(y)|<\varepsilon$ whenever $d(x, y)<\delta$ in $x, y \in K$. By 
the Gaussian estimate (3.3), we can choose a positive number $T$ so that $p(t, x, y)<\varepsilon$ for any $0<t<T$, and for any $x \in X$ and $y \in K$ satisfying $d(x, y) \geq \delta$. Then we have that, for any $x \in X$

$$
\begin{aligned}
& \left|P_{t} f(x)-f(x)\right|=\left|\int_{K} p(t, x, y) f(y) m(d y)-f(x)\right| \\
& \leq \int_{K} p(t, x, y)|f(y)-f(x)| m(d y) \\
& =\int_{B_{\delta}(x) \cap K} p(t, x, y)|f(y)-f(x)| m(d y)+\int_{\left.\left(B_{\delta}(x)\right)^{c} \cap K\right)} p(t, x, y)|f(y)-f(x)| m(d y) \\
& \leq \varepsilon+2 \varepsilon\|f\|_{\infty} .
\end{aligned}
$$

Thus we have shown that (F-2) holds.

\section{Examples}

In this section, several specific examples satisfying Assumption 1.1 or the assumption in Theorem 1.7 are given. In the first subsection, we explain weighted Riemannian manifolds whose weighted Ricci curvature is bounded below, and their pmG limit spaces. In the second subsection, we explain Alexandrov spaces, which are a generalization of the lower sectional curvature bound to metric spaces. In the third subsection, we give Hilbert spaces with log-concave probability measures.

\subsection{Weighted Riemanniam manifolds and pmG limit spaces}

Let $\left\{\left(M_{n}, g_{n}, w_{n}, \bar{x}_{n}\right)\right\}_{n \in \mathbb{N}}$ be a sequence of pointed complete and connected weighted $N$-dimensional Riemannian manifolds whose weight satisfies $w_{n}=e^{-V_{n}}$ for a twice continuously differentiable function $V_{n} \in C^{2}\left(M_{n}\right)$. We write the corresponding pointed metric measure space $\mathcal{M}_{n}=\left(M_{n}, d_{g_{n}}, w_{n} \mathrm{Vol}_{n}, \bar{x}_{n}\right)$ whereby $d_{g_{n}}$ denotes the distance function associated with the Riemannian metric $g_{n} ; \mathrm{Vol}_{n}$ denotes the Riemannian volume measure; and $\bar{x}_{n} \in M_{n}$ is a fixed point. Let the weighted Ricci curvature Ric $\mathcal{M}_{n}$ of $\mathcal{M}_{n}$ be bounded from below by $K$ : there exists $K \in \mathbb{R}$ so that

$$
\operatorname{Ric}_{\mathcal{M}_{n}}=\operatorname{Ric}_{g}+\nabla^{2} V_{n} \geq K g_{n},
$$

whereby $\operatorname{Ric}_{g_{n}}$ means the Ricci curvature of $\left(M_{n}, g_{n}\right)$ and $\nabla^{2}$ means the Hessian. Then $\mathcal{M}_{n}$ satisfies $\operatorname{RCD}(K, \infty)$ spaces $([59,65])$. Even when $V_{n}: M_{n} \rightarrow \mathbb{R}$ is not in $C^{2}\left(M_{n}\right)$, if $\operatorname{Ric}_{g_{n}} \geq K$ and

$$
V_{n}: M_{n} \rightarrow \mathbb{R} \text { is } K^{\prime} \text {-convex (see [65]), }
$$

then $\mathcal{M}_{n}$ satisfies $\operatorname{RCD}\left(K+K^{\prime}, \infty\right)$. If, moreover,

$$
V_{n}: M_{n} \rightarrow \mathbb{R} \text { is }\left(K^{\prime}, N^{\prime}\right) \text {-convex (see [22]), }
$$

then $\mathcal{M}_{n}$ satisfies $\operatorname{RCD}^{*}\left(K+K^{\prime}, N+N^{\prime}\right)$. The Brownian motion on $M_{n}$ is a Markov process whose infinitesimal generator $A_{n}$ is

$$
A_{n}=\frac{1}{2} \Delta_{M_{n}}-\left\langle\nabla V_{n}, \nabla\right\rangle,
$$

whereby $\Delta_{M_{n}}$ is the Laplace-Beltrami operator on $M_{n}$.

If $\mathcal{M}_{n}$ satisfying $\operatorname{RCD}(K, \infty)$ (or, $\operatorname{RCD}^{*}(K, N)$ ) converges to a metric measure space $\mathcal{M}_{\infty}$ in pmG sense, then the limit space $\mathcal{M}_{\infty}$ satisfies $\operatorname{RCD}(K, \infty)$ (or, $\operatorname{RCD}^{*}(K, N)$ ), respectively (see [30, 22]). Thus we can apply our main results and obtain the weak convergence of the Brownian motions.

We have various singular examples appearing as the limit space. See e.g., [17, Example 8]. We give one of the simplest examples included in this framework. 
Example 4.1 (Collapsing: Torus $\rightarrow$ Circle). Let $\mathbb{S}^{1} \subset \mathbb{R}^{2}$ be the unit circle. Let $d_{\mathbb{S}^{1}}$ be the shortest path distance on $\mathbb{S}^{1}$, that is, the distance between $x$ and $y$ is defined by the infimum over lengths of geodesics on $\mathbb{S}^{1}$ connecting $x$ and $y$. Let

$$
\bar{H}_{\mathbb{S}^{1}}:=\frac{1}{H_{\mathbb{S}^{1}}\left(S^{1}\right)} H_{\mathbb{S}^{1}}
$$

be the normalized Hausdorff measure on $\left(\mathbb{S}^{1}, d_{\mathbb{S}^{1}}\right)$. Let $\mathbb{T}_{n}=\mathbb{S}^{1} \times \mathbb{S}^{1}$ be a two-dimensional flat torus with a metric $d_{n}=d_{\mathbb{S}^{1}} \otimes \frac{1}{n} d_{\mathbb{S}^{1}}$ and the normalized Hausdorff measure $\bar{H}_{n}$ on $\left(\mathbb{T}_{n}, d_{n}\right)$, whereby

$$
d_{\mathbb{S}^{1}} \otimes \frac{1}{n} d_{\mathbb{S}^{1}}\left(\left(x_{1}, y_{1}\right),\left(x_{2}, y_{2}\right)\right):=\sqrt{d_{\mathbb{S}^{1}}^{2}\left(x_{1}, x_{2}\right)+\frac{1}{n^{2}} d_{\mathbb{S}^{1}}^{2}\left(y_{1}, y_{2}\right)} .
$$

Then $\left(\mathbb{T}_{n}, d_{n}, \bar{H}_{n}\right)$ satisfies the $\operatorname{RCD}^{*}(0,2)$ for any $n \in \mathbb{N}$ and converges to $\left(\mathbb{S}^{1}, d_{\mathbb{S}^{1}}, \bar{H}_{\mathbb{S}^{1}}\right)$ in the measured Gromov sense. Thus we can apply our result (Theorem 1.7) and the weak convergence of the Brownian motions is equivalent to the pmG convergence of the underlying spaces.

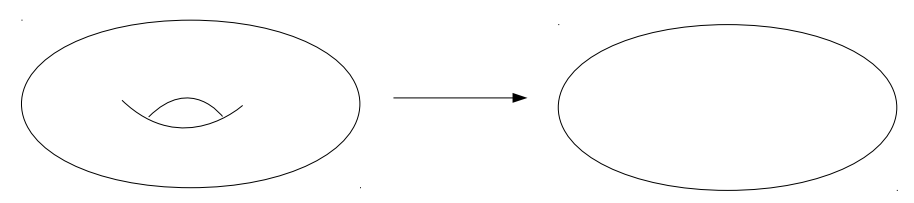

Figure 1: Tori Converge to a Circle.

\subsection{Alexandrov spaces}

We explain Alexandrov spaces, which are a generalization of lower bounds of sectional curvatures to metric spaces. We refer the reader to [15] for basic theory of Alexandrov spaces. Let $(X, d)$ be a locally compact length space. For a triple of points $p, q, r \in X$, a geodesic triangle $\triangle p q r$ is a triplet of geodesics joining each two points. Let $M^{N}(K)$ be the $N$-dimensional complete simply connected space of constant sectional curvature $K$. For a geodesic triangle $\triangle p q r$, we denote by $\triangle \widetilde{p} \widetilde{q r}$ a geodesic triangle in $M^{2}(K)$ whose corresponding edges have the same lengths as $\triangle p q r$.

A locally compact length space $(X, d)$ is said to be an Alexandrov space with Curv $\geq K$ if for every point $x \in X$, there exists an open set $U_{x}$ including $x$ so that for every geodesic triangle $\triangle p q r$ whose edges are totally included in $U_{x}$, the corresponding geodesic triangle $\triangle \widetilde{p} \widetilde{q} r$ satisfies the following condition: for every point $z \in q r$ and $\widetilde{z} \in \widetilde{q} \widetilde{r}$ with $d(q, z)=d(\widetilde{q}, \widetilde{z})$, we have

$$
d(p, z) \geq d(\widetilde{p}, \widetilde{z}) .
$$

If we consider a complete $N$-dimensional Riemannian manifold $(M, g)$, then $(M, g)$ is an Alexandrov space with Curv $\geq K$ if and only if $\sec (M) \geq K$, whereby $\sec (M)$ means the sectional curvature of $M$.

Let $\mathcal{X}=(X, d, H)$ be an $N$-dimensional Alexandrov space with Curv $\geq K$ and $H$ be the Hausdorff measure (see e.g., [15] for details). According to [55, 71], $\mathcal{X}$ satisfies $\mathrm{CD}^{*}((N-1) K, N)$. Moreover, as was shown in [42], $\mathcal{X}$ satisfies the infinitesimal Hilbertian condition, and as a result, $\mathcal{X}$ satisfies $\operatorname{RCD}^{*}((N-1) K, N)$. Thus we can apply our results (Theorem 1.2, 1.4) and if a sequence of pointed Alexandrov spaces $X_{n}$ with Curv $\geq K$ converges to the limit space $X_{\infty}$ in the pmG sense, then the Brownian motions on $X_{n}$ converge weakly to the limit Brownian motion on $X_{\infty}$. We give several examples. 
Example 4.2 (Cone $\rightarrow$ Interval). Let $X_{n} \subset \mathbb{R}^{3}$ be a cone defined by $X_{n}=\{(x, y, z) \in$ $\left.\mathbb{R}^{3}: y^{2}+z^{2}=\frac{1}{n} x, 0 \leq x<1\right\} \cup\left\{(x, y, z) \in \mathbb{R}^{3}: y^{2}+z^{2}=\frac{1}{n}, x=1\right\}$. Let $d_{n}$ be the shortest path distance on $X_{n}$ and $\bar{H}_{n}$ be the normalized Hausdorff measure on $X_{n}$. Then $\left(X_{n}, d_{n}, \bar{H}_{n}\right)$ satisfies $\operatorname{RCD}^{*}(0,2)$ and converges to $([0,1],|\cdot|, m)$ in the measured Gromov sense, whereby $m$ is a measure on $[0,1]$. Thus we can apply our result (Theorem 1.7) and the weak convergence of the Brownian motions is equivalent to the pmG convergence of the underlvina spaces.

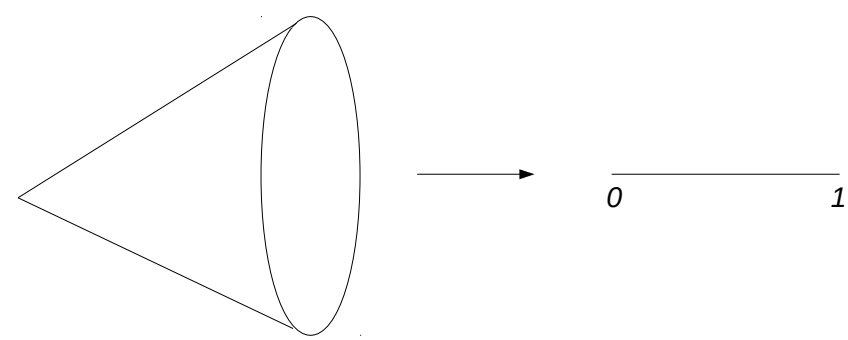

Figure 2: Cones Converge to an Interval.

As a second example, we give a sequence of polygons made by the barycentric subdivision. The limit space has dense singularities.

Example 4.3 (Dense Singularities [53, p. 632, Examples. (2)]). Let $X=(X, d)$ be a polyhedron in $\mathbb{R}^{3}$ with the shortest path metric $d$ on $X$. Then we can check whether $X$ is an Alexandrov space with Curv $\geq 0$, which is also an $\operatorname{RCD}^{*}(0,2)$ space. For any vertex $p \in X$, let $\angle(X, p)$ denote the sum of all inner angles at $p$ of faces $T^{\prime}$ 's such that $p$ is a vertex of $T$.

Now we construct a sequence of polyhedra $\left\{M_{n}\right\}_{n \in \mathbb{N}}$ inductively. Let $M_{1}$ be a tetrahedron in $\mathbb{R}^{3}$ with the barycenter $o$. Let $M_{n}$ be defined. Then we define $M_{n+1}$ as follows: Take a monotone decreasing sequence $\left\{\varepsilon_{n}\right\}_{n \in \mathbb{N}}$ so that $\varepsilon_{n} \rightarrow 0$ as $n \rightarrow \infty$ with $0<\varepsilon_{n}<1$ and $\varepsilon:=\Pi_{n=1}^{\infty}\left(1-\varepsilon_{n}\right)>0$. We take the barycentric subdivision of $M_{n}$. Keep the original vertices in $M_{n}$ in the same positions and move the new vertices generated by the barycentric subdivision outward along rays emanating from $o$ so small that, for the new polyhedra $M_{n+1}$ generated by the new and original vertices, we have

$$
2 \pi-\angle\left(M_{n+1}, p\right) \geq\left(1-\varepsilon_{n}\right)\left(2 \pi-\angle\left(M_{n}, p\right)\right),
$$

for any vertex $p \in M_{n}$. See [53, p. 632, Examples. (2)] for more details.

Let $d_{n}$ and $H_{n}$ be the shortest path distance and the Hausdorf measure on $M_{n}$. Then there exists the Hausdorff-limit of $M_{n}=\left(M_{n}, d_{n}\right)$, denoted by $M_{\infty}$. The limit space $M_{\infty}$ is a two-dimensional Alexandorv space with nonnegative curvature. In particular, $\left(M_{n}, d_{n}, H_{n}\right)$ converges to $\left(M_{\infty}, d_{\infty}, H_{\infty}\right)$ in the measured Gromov sense. The limit space $M_{\infty}$ also satisfies the $\operatorname{RCD}^{*}(0,2)$ by the stability of $\operatorname{RCD}^{*}(K, N)$ spaces under the measured Gromov convergence (see [22]). The set of singular points in $M_{\infty}$ is dense (see [53]). Since each diameter of $M_{n}$ is obviously uniformly bounded by the construction, we can apply our result (Theorem 1.7) and the weak convergence of the Brownian motions is equivalent to the pmG convergence of the underlying spaces.

\subsection{Hilbert space with log-concave measures}

In this subsection, we give a specific class of $\operatorname{RCD}(0, \infty)$ spaces, which is a Hilbert space with log-concave measures. This subsection follows [9].

Let $H$ be a separable Hilbert space, which would be a finite- or infinite-dimensional space, with an inner product $\langle\cdot, \cdot\rangle$ and the corresponding norm $\|\cdot\|$. A Borel probability 


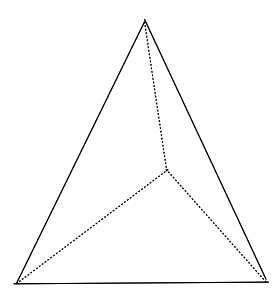

$M_{1}$

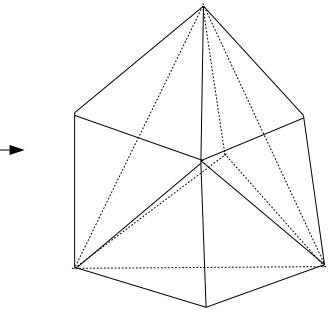

$M_{2}$

Figure 3: Polyhedra Generated by Barycentric Subdivision.

measure $\gamma$ on $H$ satisfies log-concave condition if, for all pairs of open subsets $A, B \subset H$, it holds that

$$
\log \gamma((1-t) A+t B) \geq(1-t) \log \gamma(A)+t \log \gamma(B), \quad \forall t \in[0,1] .
$$

Let $K=\operatorname{supp}[\gamma]$ and $A=A(\gamma)$ be the smallest closed linear subspace containing $K$. We write canonically

$$
A=H_{0}+h_{0}, \quad h_{0} \in K, \quad\left\|h_{0}\right\| \leq\|k\|, \quad \forall k \in K,
$$

so that $h_{0}$ is the element of the minimal norm in $K$ and $H_{0}$ is a closed linear subspace in $H$.

Let $C_{b}^{1}(A)$ be the set of all $\Phi: A \rightarrow \mathbb{R}$ which are bounded, continuous and Fréchet differentiable with a bounded continuous gradient $\nabla \Phi: A \rightarrow H_{0}$. Then, according to [9, Theorem 1.2], the following bilinear form becomes closable and the closed form becomes a symmetric quasi-regular Dirichlet form $\mathcal{E}=\mathcal{E}_{\|\cdot\|, \gamma}$ :

$$
\mathcal{E}(u, v)=\int_{K}\langle\nabla u, \nabla v\rangle_{H_{0}} d \gamma, \quad u, v \in \mathcal{F}:={\overline{C_{b}^{1}(A)}}^{\sqrt{\mathcal{E}+\|\cdot\|_{2}^{2}}} .
$$

In [9], the corresponding semigroup $\left\{P_{t}\right\}_{t \geq 0}$ associated with $(\mathcal{E}, \mathcal{F})$ satisfies $\mathrm{EVI}_{0}$ property, which is equivalent to the $\operatorname{RCD}(0, \infty)$ condition of $(H,\|\cdot\|, \gamma)$ according to [6]. Let $\left\{H_{n}=\left(H,\|\cdot\|_{n}, \gamma_{n}, \bar{x}_{n}\right)\right\}_{n \in \mathbb{N}}$ be a sequence of pointed Hilbert spaces with log-concave probability measures satisfying the above conditions. Then the weak convergence of the Brownian motions on $H_{n}$ to that on $H_{\infty}$ follows from the pmG convergence of the underlying spaces $H_{n}$ to $H_{\infty}$ (Theorem 1.2, 1.4 and [9, Theorem 1.5]).

Various infinite dimensional examples are included in the framework of Hilbert spaces with log-concave probability measures. For instance, all measures $\gamma$ of the following form satisfies the log-concave condition: let $d x$ be the Lebsgue measure on $\mathbb{R}^{N}$ and

$$
\gamma=\frac{1}{Z} e^{-V} d x \text {, whereby } V: H=\mathbb{R}^{N} \rightarrow \mathbb{R} \text { convex and } Z=\int_{\mathbb{R}^{N}} e^{-V} d x<+\infty,
$$

such as all Gaussian measures and all Gibbs measures on on a finite lattice with a convex Hamiltonian. See [9, Section 1.2] for various infinite-dimensional literatures related to stochastic partial differential equations. We give several finite-dimensional examples.

Example 4.4 ([9]). We explain several examples associated with stochastic differential equations (SDE). The first one is SDEs on the Euclidean space $\mathbb{R}^{N}$ with variable potentials. The second one is SDEs on variable convex domains in $\mathbb{R}^{N}$ with variable potentials.

(a) (SDE with Variable Convex Potentials) Let $H=\mathbb{R}^{N}$ with $1<N<\infty$. Let $\left\{V_{n}: \mathbb{R}^{N} \rightarrow \mathbb{R}\right\}_{n \in \mathbb{N}}$ be a sequence of convex functionals with a Lipschitz continuous 
gradient $\nabla V_{n}: \mathbb{R}^{N} \rightarrow \mathbb{R}^{N}$ and $\int_{\mathbb{R}^{N}} e^{-V_{n}} d x<\infty$. Take

$$
\gamma_{n}=\frac{1}{Z_{n}} e^{-V_{n}} d x, \quad \text { whereby } Z_{n}=\int_{\mathbb{R}^{N}} e^{-V_{n}} d x .
$$

Then $\gamma_{n}$ becomes a log-concave probability measure. Therefore the diffusion process associated with the Dirichlet form $\left(\mathcal{E}_{n}, \mathcal{F}_{n}\right)$ in (4.1) is a solution to the following SDE:

$$
d X_{t}^{n}=-\nabla V_{n}\left(X_{t}^{n}\right) d t+\sqrt{2} d W_{t}, \quad X_{0}=\bar{x}_{n}
$$

If $\gamma_{n}$ converges to a probability measure $\gamma_{\infty}$ weakly and $\bar{x}_{n} \rightarrow \bar{x}_{\infty}$, then $\left(\mathbb{R}^{N}, \|\right.$. $\left.\|_{2}, \gamma_{n}, \bar{x}_{n}\right)$ converges to $\left(\mathbb{R}^{N},\|\cdot\|_{2}, \gamma_{\infty}, \bar{x}_{\infty}\right)$ in the pmG sense and $\left(\mathbb{R}^{N},\|\cdot\|_{2}, \gamma_{\infty}, \bar{x}_{\infty}\right)$ is an $\operatorname{RCD}(0, \infty)$ space by the stability of the RCD property. Thus the solution to SDE (4.2) on $H_{n}=\left(H,\|\cdot\|_{n}, \gamma_{n}, \bar{x}_{n}\right)$ converges weakly to the diffusion associated with the Cheeger energy on the limit space (Theorem 1.2, 1.4 and [9, Theorem 1.5]).

(b) (SDE on Variable Convex Subsets with Variable Convex Potentials) Let $H=$ $\mathbb{R}^{N}$ with $1<N<\infty$ and $U_{n} \subset \mathbb{R}^{N}$ be a smooth convex open set. We consider a convex functional $V_{n} \in C^{1,1}\left(U_{n}\right)$ and $V_{n} \equiv+\infty$ on $\mathbb{R}^{N} \backslash U_{n}$ with $\int_{U_{n}} e^{-V_{n}} d x<\infty$ for $n \in \mathbb{N}$. Take

$$
\gamma_{n}=\left.\frac{1}{Z_{n}} e^{-V_{n}} d x\right|_{U_{n}}, \quad \text { whereby } Z_{n}=\int_{U_{n}} e^{-V_{n}} d x .
$$

Then $\gamma_{n}$ becomes a log-concave probability measure. Therefore the diffusion process associated with the Dirichlet form $\left(\mathcal{E}_{n}, \mathcal{F}_{n}\right)$ in (4.1) is a solution of the following SDE with reflection at the boundary:

$$
d X_{t}^{n}=-\nabla V_{n}\left(X_{t}^{n}\right) d t+\sqrt{2} d W_{t}+\mathbf{n}_{n}\left(X_{t}\right) d L_{t}^{n}, \quad X_{0}=\bar{x}_{n} .
$$

Here $\mathbf{n}_{n}$ is an inner normal vector to $\partial U_{n}$ and $L^{n}$ is a continuous monotone nondecreasing process which increases only when $X_{t} \in \partial U_{n}$.

If $\gamma_{n}$ converges to a probability measure $\gamma_{\infty}$ weakly and $\bar{x}_{n} \rightarrow \bar{x}_{\infty}$, then $\left(\mathbb{R}^{N}, \|\right.$. $\left.\|_{2}, \gamma_{n}, \bar{x}_{n}\right)$ converges to $\left(\mathbb{R}^{N},\|\cdot\|_{2}, \gamma_{\infty}, \bar{x}_{\infty}\right)$ in the pmG sense and $\left(\mathbb{R}^{N},\|\cdot\|_{2}, \gamma_{\infty}, \bar{x}_{\infty}\right)$ is an $\operatorname{RCD}(0, \infty)$ space by the stability of the RCD property. Thus the solution to (4.3) on $\bar{U}_{n}$ converges weakly to the diffusion associated with the Cheeger energy on the limit space (Theorem 1.2, 1.4 and [9, Theorem 1.5]).

\section{Proof of Theorem 1.2}

We first show the implication of (ii) $\Longrightarrow$ (i) in Theorem 1.2.

Proof of (ii) $\Longrightarrow$ (i) in Theorem 1.2. If we assume (ii), then it is obvious that the initial distributions $\widetilde{m}_{n}$ converge weakly to $\widetilde{m}_{\infty}$. Since the weak convergence of $\widetilde{m}_{n}$ to $\widetilde{m}_{\infty}$ is equivalent to the convergence of $m_{n}$ to $m_{\infty}$ in the sense of (2.2) (easy to check), we finish the proof of the implication (ii) $\Longrightarrow$ (i) in Theorem 1.2.

We now show the implication (i) $\Longrightarrow$ (ii).

Proof of (i) $\Longrightarrow$ (ii) in Theorem 1.2. By Definition 2.1, there exist a complete separable metric space $(X, d)$ and a family of isometric embeddings $\iota_{n}: X_{n} \rightarrow X$ such that, for any bounded continuous function $f: X \rightarrow \mathbb{R}$ with bounded support, we have

$$
\int_{X} f d\left(\iota_{n \#} m_{n}\right) \rightarrow \int_{X} f d\left(\iota_{\infty \#} m_{\infty}\right) .
$$


Set the notation for the laws of Brownian motions as follows:

$$
\mathbb{B}_{n}^{\widetilde{m}_{n}}:=\left(\iota_{n}\left(B^{n}\right), \mathbb{P}_{n}^{\widetilde{m}_{n}}\right), \quad \mathbb{B}_{n}^{\bar{x}_{n}}:=\left(\iota_{n}\left(B^{n}\right), \mathbb{P}_{n}^{\bar{x}_{n}}\right) .
$$

Hereafter we identify $\iota_{n}\left(X_{n}\right)$ with $X_{n}$ and we omit $\iota_{n}$ for simplifying the notation.

To show the weak convergence of the Brownian motions, we have two steps. The first step is to show the weak convergence of finite-dimensional distributions, and the second is to show tightness. We first show the weak convergence of finite-dimensional distributions in the case that the initial distribution is the Dirac measure $\delta_{\bar{x}_{n}}$.

Lemma 5.1 (Convergence of Finite-Dimensional Distributions). For any $k \in \mathbb{N}, 0=t_{0}<$ $t_{1}<t_{2}<\cdots<t_{k}<\infty$ and $f_{1}, f_{2}, \ldots, f_{k} \in C_{b}(X)$, the following holds:

$$
\mathbb{E}^{\bar{x}_{n}}\left[f_{1}\left(B_{t_{1}}^{n}\right) \cdots f_{k}\left(B_{t_{k}}^{n}\right)\right]^{n \rightarrow \infty} \mathbb{E}^{\bar{x}_{\infty}}\left[f_{1}\left(B_{t_{1}}^{\infty}\right) \cdots f_{k}\left(B_{t_{k}}^{\infty}\right)\right] .
$$

Proof. Since the limit Brownian motion $\mathbb{B}_{\infty}^{\bar{x}_{\infty}}$ is conservative, it suffices to show the statement only for $f_{1}, f_{2}, \ldots, f_{k} \in C_{b}(X) \cap L^{2}\left(X ; m_{\infty}\right)$. In fact, for any $\varepsilon>0$ and $T>0$, there exists $R=R(\varepsilon, T)$ so that the open ball $B_{R}\left(\bar{x}_{\infty}\right)$ satisfies

$$
\mathbb{E}^{\bar{x} \infty} \mathbf{1}_{B_{R}\left(\bar{x}_{\infty}\right)}\left(B_{t}^{\infty}\right)=\mathbb{P}^{\bar{x}_{\infty}}\left(B_{t}^{\infty} \in B_{R}\left(\bar{x}_{\infty}\right)\right) \geq 1-\varepsilon, \quad \forall t \in[0, T] .
$$

If we know that $\mathbb{E}^{\bar{x}_{n}}\left(f\left(B_{t}^{n}\right)\right)$ converges to $\mathbb{E}^{\bar{x}_{\infty}}\left(f\left(B_{t}^{\infty}\right)\right)$ for any $f \in C_{b}(X) \cap L^{2}\left(X ; m_{\infty}\right)$, then we know that

$$
\lim _{n \rightarrow \infty} \mathbb{P}^{\bar{x}_{n}}\left(B_{t}^{n} \in B_{R}\left(\bar{x}_{\infty}\right)\right)=\lim _{n \rightarrow \infty} \mathbb{E}^{\bar{x}_{n}}\left(\mathbf{1}_{B_{R}\left(\bar{x}_{\infty}\right)}\left(B_{t}^{n}\right)\right)=\mathbb{E}^{\bar{x}_{\infty}}\left(\mathbf{1}_{B_{R}\left(\bar{x}_{\infty}\right)}\left(B_{t}^{\infty}\right)\right) \geq 1-\varepsilon,
$$

for any $t \in[0, T]$. Therefore, for any $f_{1}, \ldots, f_{k} \in C_{b}(X)$, and any small $\delta>0$, we can choose $R>0$ large enough so that

$$
\begin{aligned}
& \lim _{n \rightarrow \infty} \mathbb{E}^{\bar{x}_{n}}\left(f_{1}\left(B_{t_{1}}^{n}\right) \cdots f_{k}\left(B_{t_{k}}^{n}\right)\right) \\
& =\lim _{n \rightarrow \infty} \mathbb{E}^{\bar{x}_{n}}\left(f_{1}\left(B_{t_{1}}^{n}\right) \cdots f_{k}\left(B_{t_{k}}^{n}\right): \bigcap_{j=1}^{k}\left\{B_{t_{j}}^{n} \in B_{R}\left(\bar{x}_{\infty}\right)\right\}\right) \\
& \quad \quad+\lim _{n \rightarrow \infty} \mathbb{E}^{\bar{x}_{n}}\left(f_{1}\left(B_{t_{1}}^{n}\right) \cdots f_{k}\left(B_{t_{k}}^{n}\right):\left(\bigcap_{j=1}^{k}\left\{B_{t_{j}}^{n} \in B_{R}\left(\bar{x}_{\infty}\right)\right\}\right)^{c}\right) \\
& =\lim _{n \rightarrow \infty} \mathbb{E}^{\bar{x}_{n}}\left(f_{1} \mathbf{1}_{B_{R}}\left(B_{t_{1}}^{n}\right) \cdots f_{k} \mathbf{1}_{B_{R}}\left(B_{t_{k}}^{n}\right)\right)+\delta .
\end{aligned}
$$

Thus we may show the proof only for $f_{1}, f_{2}, \ldots, f_{k} \in C_{b}(X) \cap L^{2}\left(X ; m_{\infty}\right)$.

Recall that we have the following equality (see Subsection 3.1): for every $f \in$ $C_{b}(X) \cap L^{2}\left(X ; m_{\infty}\right)$,

$$
\mathbb{E}_{n}^{x}\left(f\left(B_{t}^{n}\right)\right)=P_{t}^{n} f(x)
$$

for every $x \in X_{n}$. Here recall that $\left\{P_{t}^{n}\right\}_{t \geq 0}$ is the semigroup defined in (3.1) by the action of the heat flow whereby $P_{t}$ is a modification of the heat semigroup $H_{t}$ and $P_{t}^{n} f(x)$ can be defined for every point $x \in X^{n}$ if $f \in C_{b}(X) \cap L^{2}\left(X ; m_{\infty}\right)$. Since the Brownian motion $\left(\left\{\mathbb{P}_{n}^{x}\right\}_{x \in X_{n}},\left\{B_{t}^{n}\right\}_{t \geq 0}\right)$ is constructed by the Kolmogorov extension theorem with the integral kernel $p_{n}(t, x, d y)$ of $\left\{P_{t}^{n}\right\}_{t \geq 0}$ as in Section 3.1, the equality (5.1) holds for every point $x \in X_{n}$.

By using the Markov property, for all $n \in \overline{\mathbb{N}}$, we have

$$
\begin{aligned}
& \mathbb{E}_{n}^{\bar{x}_{n}}\left[f_{1}\left(B_{t_{1}}^{n}\right) \cdots f_{k}\left(B_{t_{k}}^{n}\right)\right] \\
& =P_{t_{1}-t_{0}}^{n}\left(f_{1} P_{t_{2}-t_{1}}^{n}\left(f_{2} \cdots P_{t_{k}-t_{k-1}}^{n} f_{k}\right)\right)\left(\bar{x}_{n}\right) \\
& =: \mathcal{P}_{k}^{n}\left(\bar{x}_{n}\right) .
\end{aligned}
$$


By [3, Theorem 7.3], $\mathcal{P}_{k}^{n}$ is bounded Lipschitz on $X_{n}$ whose Lipschitz constant depends only on the curvature lower-bound $K$.

For later arguments, we extend $\mathcal{P}_{k}^{n}$ to the whole space $X$ (note that $\mathcal{P}_{k}^{n}$ is defined only on each $X_{n}$ ). The key point is to extend $\mathcal{P}_{k}^{n}$ to the whole space $X$ preserving its Lipschitz regularity and bounds.

Proposition 5.2. ([50, Corollary 1,2]) Let $\widetilde{\mathcal{P}}_{k}^{n}$ be the function defined on the whole space $X$ as follows:

$$
\widetilde{\mathcal{P}_{k}^{n}}(x):=\left(\sup _{a \in X_{n}}\left\{\mathcal{P}_{k}^{n}(a)-H d(a, x)\right\} \wedge \sup _{a \in X_{n}} \mathcal{P}_{k}^{n}(a)\right) \vee \inf _{a \in X_{n}} \mathcal{P}_{k}^{n}(a), \quad x \in X .
$$

Here $H$ denotes the same Lipschitz constant of the original function $\mathcal{P}_{k}^{n}$. Then $\widetilde{\mathcal{P}_{k}^{n}}$ is a bounded Lipschitz continuous function on the whole space $X$ with the same Lipschitz constant $H$ and the same bound. Moreover $\widetilde{\mathcal{P}_{k}^{n}}=\mathcal{P}_{k}^{n}$ on the original domain $X_{n}$. The function $\widetilde{\mathcal{P}}_{k}^{n}$ is called McShane extension of $\mathcal{P}_{k}^{n}$.

We now return to the proof of Lemma 5.1. We have that

$$
\begin{aligned}
& \left|\mathbb{E}_{n}^{\bar{x}_{n}}\left[f_{1}\left(B_{t_{1}}^{n}\right) \cdots f_{k}\left(B_{t_{k}}^{n}\right)\right]-\mathbb{E}_{n}^{\bar{x}_{\infty}}\left[f_{1}\left(B_{t_{1}}^{\infty}\right) \cdots f_{k}\left(B_{t_{k}}^{\infty}\right)\right]\right| \\
& =\left|\mathcal{P}_{k}^{n}\left(\bar{x}_{n}\right)-\mathcal{P}_{k}^{\infty}\left(\bar{x}_{\infty}\right)\right| \\
& \leq\left|\mathcal{P}_{k}^{n}\left(\bar{x}_{n}\right)-\widetilde{\mathcal{P}_{k}^{n}}\left(\bar{x}_{\infty}\right)\right|+\left|\widetilde{\mathcal{P}_{k}^{n}}\left(\bar{x}_{\infty}\right)-\mathcal{P}_{k}^{\infty}\left(\bar{x}_{\infty}\right)\right| \\
& =:(\mathrm{I})_{n}+(\mathrm{II})_{n} \text {. }
\end{aligned}
$$

Therefore it suffices to show (I) $)_{n} \rightarrow 0$ and (II) $)_{n} \rightarrow 0$ as $n \rightarrow \infty$.

We first discuss to show $(\mathrm{I})_{n} \rightarrow 0$. Since $\left\|P_{t}^{n} f\right\|_{\infty}=\|f\|_{\infty}\left\|\int_{X_{n}} p_{n}(t, x, y) m_{n}(d y)\right\|_{\infty} \leq$ $\|f\|_{\infty}$, for any $f \in C_{b}\left(X_{n}\right) \cap L^{2}\left(X ; m_{\infty}\right)$, we have

$$
\sup _{n \in \mathbb{N}}\left\|\mathcal{P}_{k}^{n}\right\|_{\infty} \leq \prod_{i=1}^{k}\left\|f_{i}\right\|_{\infty}<\infty .
$$

Therefore, by Proposition 5.2, it holds that

$$
\sup _{n \in \mathbb{N}}\left\|\widetilde{\mathcal{P}_{k}^{n}}\right\|_{\infty}<\infty
$$

By [3, Theorem 7.3], we have that $\operatorname{Lip}_{X}\left(P_{t}^{n} f\right) \leq C(t, K)\|f\|_{\infty}$ for any $f \in L^{\infty}\left(X_{n} ; m_{n}\right) \cap$ $L^{2}\left(X ; m_{\infty}\right)$ for some positive $C(t, K)$ depending only on $t, K$. Here $\operatorname{Lip}_{X}(f)$ means the global Lipschitz constant of a Lipschitz function $f$ on $X$. Thus by considering (5.3), there exists a constant $L$ depending only on $t_{k}, K$ and $\left\|f_{1}\right\|_{\infty}, \ldots,\left\|f_{k}\right\|_{\infty}$ (but independent of $n$ ) so that

$$
\sup _{n \in \mathbb{N}} \operatorname{Lip}_{X}\left(\mathcal{P}_{k}^{n}\right) \leq \sup _{n \in \mathbb{N}} C\left(t_{k}, K\right)\left\|f_{k} \mathcal{P}_{k-1}^{n}\right\|_{\infty}<L<\infty
$$

By the property of the McShane extension in Proposition 5.2, we have that

$$
\sup _{n \in \mathbb{N}} \operatorname{Lip}_{X}\left(\widetilde{\mathcal{P}_{k}^{n}}\right) \leq \sup _{n \in \mathbb{N}} C\left(t_{k}, K\right)\left\|f_{k} \mathcal{P}_{k-1}^{n}\right\|_{\infty}<L<\infty
$$

Thus we have

$$
\begin{aligned}
(\mathrm{I})_{n}=\left|\mathcal{P}_{k}^{n}\left(\bar{x}_{n}\right)-\widetilde{\mathcal{P}_{k}^{n}}\left(\bar{x}_{\infty}\right)\right| & =\left|\widetilde{\mathcal{P}_{k}^{n}}\left(\bar{x}_{n}\right)-\widetilde{\mathcal{P}_{k}^{n}}\left(\bar{x}_{\infty}\right)\right| \\
& \leq \operatorname{Lip}\left(\widetilde{\mathcal{P}_{k}^{n}}\right) d\left(\bar{x}_{n}, \bar{x}_{\infty}\right) \\
& \leq \operatorname{Ld}\left(\bar{x}_{n}, \bar{x}_{\infty}\right) \\
& \rightarrow 0 \quad(n \rightarrow \infty) .
\end{aligned}
$$


We next show that (II) $)_{n} \rightarrow 0$. By (5.4) and (5.5), we can apply the Ascoli-Arzelá theorem to $\left\{\widetilde{\mathcal{P}_{k}^{n}}\right\}_{n \in \mathbb{N}}$ so that $\left\{\widetilde{\mathcal{P}_{k}^{n}}\right\}_{n \in \mathbb{N}}$ is relatively compact. Therefore, for any subsequence $\left\{\widetilde{\mathcal{P}_{k}^{n^{\prime}}}\right\}_{\left\{n^{\prime}\right\}}$ whereby $\left\{n^{\prime}\right\} \subset\{n\}$, there exists a further subsequence $\left.\widetilde{\mathcal{P}_{k}^{n^{\prime \prime}}}\right\}_{\left\{n^{\prime \prime}\right\}}$ whereby $\left\{n^{\prime \prime}\right\} \subset\left\{n^{\prime}\right\}$ satisfying

$$
\widetilde{\mathcal{P}_{k}^{n^{\prime \prime}}} \rightarrow F^{\prime \prime} \text { uniformly in } X \text {. }
$$

On the other hand, we have that $\mathcal{P}_{k}^{n}$ converges to $\mathcal{P}_{k}^{\infty} L^{2}$-strongly in the sense of Definition 2.10. We give a proof below.

Lemma 5.3. $\mathcal{P}_{k}^{n}$ converges to $\mathcal{P}_{k}^{\infty}$ in the $L^{2}$-strong sense in Definition 2.10.

Proof. By Theorem 2.11, the statement is true for $k=1$. Assume that the statement is true when $k=l$. Since we have

$$
\mathcal{P}_{l+1}^{n}=P_{t_{l+1}-t_{l}}^{n}\left(f_{l+1}^{(n)} \mathcal{P}_{l}^{n}\right)
$$

by Theorem 2.11, it is sufficient to show $f_{l+1} \mathcal{P}_{l}^{n} \rightarrow f_{l+1} \mathcal{P}_{l}^{\infty}$ strongly in $L^{2}$. This is obvious to be true because $\mathcal{P}_{l}^{n} \rightarrow \mathcal{P}_{l}^{\infty}$ strongly (the assumption of the induction), $f_{l+1} \in C_{b}(X)$ and $\mathcal{P}_{l}^{n}$ is bounded uniformly in $n$ thanks to (5.4). Thus the statement is true for any $k \in \mathbb{N}$.

We return to the proof of Lemma 5.1.

Proof of Lemma 5.1. By using Lemma 5.3 and (5.6), it is obvious to check that

$$
\left.F^{\prime \prime}\right|_{X_{\infty}}=\mathcal{P}_{k}^{\infty},
$$

whereby $\left.F^{\prime \prime}\right|_{X_{\infty}}$ means the restriction of $F^{\prime \prime}$ into $X_{\infty}$. The R.H.S. $\mathcal{P}_{k}^{\infty}$ of the above equality is clearly independent of choices of subsequences and thus the limit $\left.F^{\prime \prime}\right|_{X_{\infty}}$ is independent of choices of subsequences. Thus we conclude that

$$
\widetilde{\mathcal{P}_{k}^{n}} \rightarrow \mathcal{P}_{k}^{\infty} \quad \text { uniformly in } X_{\infty} .
$$

Now we return to show (II) ${ }_{n}$ goes to zero. By (5.7), we have that

$$
\begin{aligned}
(\mathrm{II})_{n}=\left|\widetilde{\mathcal{P}_{k}^{n}}\left(\bar{x}_{\infty}\right)-\mathcal{P}_{k}^{\infty}\left(\bar{x}_{\infty}\right)\right| & \leq\left\|\widetilde{\mathcal{P}_{k}^{n}}-\mathcal{P}_{k}^{\infty}\right\|_{\infty, X_{\infty}} \\
& \rightarrow 0 \quad(n \rightarrow \infty) .
\end{aligned}
$$

Here $\|\cdot\|_{\infty, X_{\infty}}$ means the uniform norm on $X_{\infty}$. Thus we finish the proof of Lemma 5.1 .

We next show the weak convergence of finite-dimensional distributions for the case that initial distributions are $W_{1}$-convergent, which includes $\widetilde{m}_{n}$ for the case of $m_{n}\left(X_{n}\right)=$ $\infty$.

Lemma 5.4. Let $\left\{\nu_{n}\right\}_{n \in \mathbb{N}} \subset \mathcal{P}\left(X_{n}\right)$ be a sequence of probability measures on $X_{n} \subset X$ converging to $\nu_{\infty} \in \mathcal{P}\left(X_{\infty}\right)$ in $W_{1}$-distance. Then, for any $k \in \mathbb{N}, 0=t_{0}<t_{1}<t_{2}<\cdots<$ $t_{k}<\infty$ and $f_{1}, f_{2}, \ldots, f_{k} \in C_{b}(X) \cap L^{2}\left(X ; m_{\infty}\right)$, the following holds:

$$
\mathbb{E}^{\nu_{n}}\left[f_{1}\left(B_{t_{1}}^{n}\right) \cdots f_{k}\left(B_{t_{k}}^{n}\right)\right] \stackrel{n \rightarrow \infty}{\rightarrow} \mathbb{E}^{\nu_{\infty}}\left[f_{1}\left(B_{t_{1}}^{\infty}\right) \cdots f_{k}\left(B_{t_{k}}^{\infty}\right)\right] .
$$

Proof. By the same argument at the beginning of Lemma 5.1, it suffices to show the statement for any $f_{1}, f_{2}, \ldots, f_{k} \in C_{b}(X) \cap L^{2}\left(X ; m_{\infty}\right)$. Recall that we set in Lemma 5.1 as follows:

$$
\begin{aligned}
& \mathbb{E}_{n}^{x}\left[f_{1}\left(B_{t_{1}}^{n}\right) \cdots f_{k}\left(B_{t_{k}}^{n}\right)\right] \\
& =P_{t_{1}-t_{0}}^{n}\left(f_{1}^{(n)} P_{t_{2}-t_{1}}^{n}\left(f_{2}^{(n)} \cdots P_{t_{k}-t_{k-1}}^{n} f_{k}^{(n)}\right)\right)(x) \\
& =: \mathcal{P}_{k}^{n}(x) .
\end{aligned}
$$


By the Kantorovich-Rubinstein duality (see e.g., [70, Theorem 5.10]), we have

$$
W_{1}\left(\nu_{n}, \nu_{\infty}\right)=\frac{1}{L} \sup \left\{\int_{X} f d \nu_{n}-\int_{X} f \nu_{\infty}: f \in \operatorname{Lip}_{b}(X), \operatorname{Lip}_{X}(f) \leq L\right\} .
$$

According to (5.4) and (5.5), we have that $\widetilde{\mathcal{P}_{k}^{n}}$ is bounded and $\sup _{n \in \mathbb{N}} \operatorname{Lip}\left(\widetilde{\mathcal{P}_{k}^{n}}\right)<L<\infty$ for some constant $L$. Thus we have that

$$
\left|\int_{X} \widetilde{\mathcal{P}}_{k}^{n} d \nu_{n}-\int_{X} \widetilde{\mathcal{P}}_{k}^{n} d \nu_{\infty}\right| \leq L W_{1}\left(\nu_{n}, \nu_{\infty}\right) .
$$

Since $\widetilde{\mathcal{P}_{k}^{n}}$ converges to $\mathcal{P}_{k}^{\infty}$ uniformly in $C_{b}\left(X_{\infty}\right)$ by (5.7), and $\nu_{n}$ converges to $\nu_{\infty}$ in the $W_{1}$-distance, by using (5.8), we have that

$$
\begin{aligned}
& \left|\mathbb{E}^{\nu_{n}}\left[f_{1}\left(B_{t_{1}}^{n}\right) \cdots f_{k}\left(B_{t_{k}}^{n}\right)\right]-\mathbb{E}^{\nu_{\infty}}\left[f_{1}\left(B_{t_{1}}^{\infty}\right) \cdots f_{k}\left(B_{t_{k}}^{\infty}\right)\right]\right| \\
& =\left|\int_{X} \mathcal{P}_{k}^{n} d \nu_{n}-\int_{X} \mathcal{P}_{k}^{\infty} d \nu_{\infty}\right| \\
& \leq\left|\int_{X} \mathcal{P}_{k}^{n} d \nu_{n}-\int_{X} \widetilde{\mathcal{P}_{k}^{n}} d \nu_{\infty}\right|+\left|\int_{X} \widetilde{\mathcal{P}_{k}^{n}} d \nu_{\infty}-\int_{X} \mathcal{P}_{k}^{\infty} d \nu_{\infty}\right| \\
& \leq L W_{1}\left(\nu_{n}, \nu_{\infty}\right)+\left\|\widetilde{\mathcal{P}_{k}^{n}}-\mathcal{P}_{k}^{n}\right\|_{\infty, X} \int_{X} d \nu_{\infty} \\
& \rightarrow 0, \quad n \rightarrow \infty .
\end{aligned}
$$

Thus we have completed the proof.

We now show the weak convergence of finite-dimensional distributions for the case that initial distributions are $\frac{1}{m_{n}\left(X_{n}\right)} m_{n}$, which corresponds to the case of $m_{n}\left(X_{n}\right)<\infty$.

Lemma 5.5. Let $m_{n}\left(X_{n}\right)<\infty$ for any $n \in \mathbb{N}$. Then, for any $k \in \mathbb{N}, 0=t_{0}<t_{1}<t_{2}<$ $\cdots<t_{k}<\infty$ and $f_{1}, f_{2}, \ldots, f_{k} \in C_{b}(X)$, the following holds:

$$
\mathbb{E}^{\widetilde{m}_{n}}\left[f_{1}\left(B_{t_{1}}^{n}\right) \cdots f_{k}\left(B_{t_{k}}^{n}\right)\right] \stackrel{n \rightarrow \infty}{\rightarrow} \mathbb{E}^{\widetilde{m}_{\infty}}\left[f_{1}\left(B_{t_{1}}^{\infty}\right) \cdots f_{k}\left(B_{t_{\infty}}^{\infty}\right)\right] .
$$

Proof. Because of $m_{n}\left(X_{n}\right)<\infty$, we have $f \in L^{2}\left(X, m_{n}\right)$ for all $f \in C_{b}(X)$ for any $n \in \overline{\mathbb{N}}$. Since $\widetilde{m}_{n}$ converges weakly to $\widetilde{m}_{\infty}$ in $\mathcal{P}(X)$, for any $\varepsilon>0$, there exists a compact set $K \subset X$ so that

$$
\sup _{n \in \mathbb{N}} \widetilde{m}_{n}\left(K^{c}\right)<\varepsilon
$$

Thus, by (5.3), for any $\delta>0$, there exists a compact set $K \subset X$ so that

$$
\sup _{n \in \mathbb{N}}\left|\int_{X_{n}} \mathcal{P}_{k}^{n} d \widetilde{m}_{n}-\int_{K} \mathcal{P}_{k}^{n} d \widetilde{m}_{n}\right| \leq\left(\prod_{i=1}^{k}\left\|f_{i}\right\|_{\infty}\right) \sup _{n \in \mathbb{N}} \widetilde{m}_{n}\left(K^{c}\right)<\delta .
$$

Take $r>0$ so that $K \subset B_{r}\left(\bar{x}_{n}\right):=\left\{x \in X: d\left(\bar{x}_{n}, x\right)<r\right\}$. Let $\widetilde{\mathbf{1}}_{r}^{R}$ denote the following function: $(r<R)$

$$
\widetilde{\mathbf{1}}_{r}^{R}(x)= \begin{cases}1, & x \in B_{r}\left(\bar{x}_{n}\right), \\ 1-\frac{d\left(x, B_{r}\left(\bar{x}_{n}\right)\right)}{R-r}, & x \in B_{R}\left(\bar{x}_{n}\right) \backslash B_{r}\left(\bar{x}_{n}\right), \\ 0, & \text { otherwise }\end{cases}
$$


Then $\widetilde{\mathbf{1}}_{r}^{R} \in C_{b s}(X)$. Thus, by Theorem 2.11 and (5.9), for any $\delta>0$, there exists $r>0$ so that

$$
\begin{aligned}
& \left|\mathbb{E}^{\widetilde{m}_{n}}\left[f_{1}\left(B_{t_{1}}^{n}\right) \cdots f_{k}\left(B_{t_{k}}^{n}\right)\right]-\mathbb{E}^{\widetilde{m}_{\infty}}\left[f_{1}\left(B_{t_{1}}^{\infty}\right) \cdots f_{k}\left(B_{t_{\infty}}^{\infty}\right)\right]\right| \\
= & \left|\int_{X_{n}} \mathcal{P}_{k}^{n} d \widetilde{m}_{n}-\int_{X_{\infty}} \mathcal{P}_{k}^{\infty} d \widetilde{m}_{\infty}\right| \\
= & \mid \int_{X_{n}} \mathcal{P}_{k}^{n} d \widetilde{m}_{n}-\int_{X_{n}} \widetilde{\mathbf{1}}_{r}^{R} \mathcal{P}_{k}^{n} d \widetilde{m}_{n}+\int_{X_{n}} \widetilde{\mathbf{1}}_{r}^{R} \mathcal{P}_{k}^{n} d \widetilde{m}_{n} \\
& -\int_{X_{n}} \widetilde{\mathbf{1}}_{r}^{R} \mathcal{P}_{k}^{\infty} d \widetilde{m}_{\infty}+\int_{X_{n}} \widetilde{\mathbf{1}}_{r}^{R} \mathcal{P}_{k}^{\infty} d \widetilde{m}_{\infty}-\int_{X_{\infty}} \mathcal{P}_{k}^{\infty} d \widetilde{m}_{\infty} \mid \\
\leq \delta & \left|\int_{X} \widetilde{\mathbf{1}}_{r}^{R} \mathcal{P}_{k}^{n} d \widetilde{m}_{n}-\int_{X} \widetilde{\mathbf{1}}_{r}^{R} \mathcal{P}_{k}^{\infty} d \widetilde{m}_{\infty}\right|+\delta \\
& \stackrel{n \rightarrow \infty}{\rightarrow} 2 \delta .
\end{aligned}
$$

In the fourth line, the first $\delta$ comes from using (5.9) and the second $\delta$ comes from using the tightness of the single measure $m_{\infty}$. The the middle term in the fourth line converges to zero thanks to the $L^{2}$-strong convergence of the heat semigroup $P_{t}$ in the sense of Definition 2.10. Note that the total mass $m_{n}\left(X_{n}\right) \rightarrow m_{\infty}\left(X_{\infty}\right)(\leq \infty)$ because of the pmG convergence. Thus we have completed the proof.

Now we show the tightness of $\left\{\mathbb{B}^{\widetilde{m}_{n}}\right\}$. For later arguments, we show the tightness for more general initial distributions $\nu_{n}$ than $\widetilde{m}_{n}$.

Lemma 5.6. Let $\nu_{n} \in \mathcal{P}\left(X_{n}\right)$ satisfy the following conditions:

(i) $\nu_{n} \rightarrow \nu_{\infty}$ weakly in $\mathcal{P}(X)$;

(ii) $\nu_{n}$ is absolutely continuous with respect to $m_{n}$ with $d \nu_{n}=\phi_{n} d m_{n}$ and there exists a positive constant $M$ so that, for any $r>0$,

$$
\sup _{n \in \mathbb{N}}\left\|\phi_{n}\right\|_{\infty, B_{r}\left(\bar{x}_{n}\right)}<M<\infty .
$$

Then $\left\{\mathbb{B}^{\nu_{n}}\right\}_{n \in \mathbb{N}}$ is tight in $\mathcal{P}(C([0, \infty), X))$.

Proof. Let us denote the law of $h\left(B^{n}\right)$ for $h \in \operatorname{Lip}_{b}(X)$ as follows:

$$
\mathbb{B}^{\nu_{n}, h}=\left(h\left(B^{n}\right), \mathbb{P}_{n}^{\nu_{n}}\right) .
$$

It is easy to show that $\operatorname{Lip}_{b}(X)$ strongly separates points in $C_{b}(X)$, that is, for every $x$ and $\varepsilon>0$, there exists a finite set $\left\{h_{i}\right\}_{i=1}^{l} \subset \operatorname{Lip}_{b}(X)$ so that

$$
\inf _{y: d(y, x) \geq \varepsilon} \max _{1 \leq i \leq l}\left|h_{i}(x)-h_{i}(y)\right|>0 .
$$

Therefore, by [23, Corollary 3.9.2] with Lemma 5.4, the following two statements are equivalent:

(i) $\left\{\mathbb{B}^{\nu_{n}}\right\}_{n \in \mathbb{N}}$ is tight in $\mathcal{P}(C([0, \infty), X))$;

(ii) $\left\{\mathbb{B}^{\nu_{n}, h}\right\}_{n \in \mathbb{N}}$ is tight in $\mathcal{P}(C([0, \infty), \mathbb{R}))$.

Thus we will show that, for any $h \in \operatorname{Lip}_{b}(X)$,

$$
\left\{\mathbb{B}^{\nu_{n}, h}\right\}_{n \in \mathbb{N}} \quad \text { is tight in } \quad \mathcal{P}(C([0, \infty) ; \mathbb{R})) .
$$

We note that, although [23, Corollary 3.9.2] gives sufficient conditions for tightness only in the càdlàg space $D([0, \infty) ; X)$, since the law of each Brownian motion $\mathbb{B}_{n}^{\tilde{m}_{n}}$ for 
$n \in \overline{\mathbb{N}}$ has its support on the space of continuous paths $C([0, \infty) ; X)$, the tightness in $D([0, \infty) ; X)$ implies the tightness in $C([0, \infty), X)$. See, e.g., [24, Lemma 5 in Appendix] for this point.

Since $\nu_{n}$ converges weakly to $\nu_{\infty}$ in $\mathcal{P}(X)$, the set of the laws of the initial distributions $\left\{\left(h\left(B_{0}^{n}\right), \mathbb{P}_{n}^{\nu_{n}}\right)\right\}_{n \in \mathbb{N}}=\left\{h_{\#} \widetilde{m}_{n}\right\}_{n \in \mathbb{N}}$ is clearly tight in $\mathcal{P}(\mathbb{R})$. For $\delta>0$, let us define

$$
L_{\eta, T}^{n, h}(x):=\mathbb{P}_{n}^{x}\left(\sup _{\substack{0 \leq s, t \leq T \\|t-s| \leq \eta}}\left|h\left(B_{t}\right)-h\left(B_{s}\right)\right|>\delta\right) .
$$

The desired result we would like to show is the following:

$$
\lim _{\eta \rightarrow 0} \sup _{n \in \mathbb{N}} \int_{X_{n}} L_{\eta, T}^{n, h} d \nu_{n}=0
$$

for any $T>0$. By conditions (i) and (ii) in this lemma, for any $\varepsilon>0$, there exists $R>0$ so that

$$
\begin{aligned}
\int_{X_{n}} L_{\eta, T}^{n, h} d \nu_{n} & =\left\|\phi_{n} \mathbf{1}_{B_{R}\left(\bar{x}_{n}\right)}\right\|_{\infty} \int_{X_{n}} L_{\eta, T}^{n, h} \mathbf{1}_{B_{R}\left(\bar{x}_{n}\right)} d m_{n}+\nu_{n}\left(B_{R}^{c}\left(\bar{x}_{n}\right)\right) \\
& <M \int_{X_{n}} L_{\eta, T}^{n, h} \mathbf{1}_{B_{R}\left(\bar{x}_{n}\right)} d m_{n}+\varepsilon .
\end{aligned}
$$

It suffices to show, for any $T, R>0$,

$$
\lim _{\eta \rightarrow 0} \sup _{n \in \mathbb{N}} \int_{X_{n}} L_{\eta, T}^{n, h} \mathbf{1}_{B_{R}\left(\bar{x}_{n}\right)} d m_{n}=0 .
$$

Let $m_{n, R}:=\mathbf{1}_{Y_{n}^{R}} m_{n}$ whereby

$$
Y_{n}^{R}=\overline{B_{R}\left(\bar{x}_{n}\right)}
$$

is the closure of the open ball $B_{R}\left(\bar{x}_{n}\right)$. We have

$$
\begin{aligned}
\int_{X_{n}} L_{\eta, T}^{n, h} d m_{n, R}= & \mathbb{P}_{n, R+r}^{m_{n, R}}\left(\sup _{\substack{0 \leq s, t \leq T \\
|t-s| \leq \eta}}\left|h\left(B_{t}^{n}\right)-h\left(B_{s}^{n}\right)\right|>\delta: \Lambda_{r}\right) \\
& +\mathbb{P}^{m_{n, R}}\left(\sup _{\substack{0 \leq s, t \leq T \\
|t-s| \leq \eta}}\left|h\left(B_{t}^{n}\right)-h\left(B_{s}^{n}\right)\right|>\delta: \Lambda_{r}^{c}\right) \\
:=(\mathrm{I})_{\mathrm{n}, \eta}+(\mathrm{II})_{\mathrm{n}, \eta}, &
\end{aligned}
$$

whereby $\Lambda_{r}:=\left\{w \in \Omega^{n}: \sup _{0 \leq t \leq T}\left|d_{n}\left(B_{t}^{n}, \bar{x}_{n}\right)-d_{n}\left(B_{0}^{n}, \bar{x}_{n}\right)\right|<r\right\}$. Here $\mathbb{P}_{n, r}^{x}$ is a conservative diffusion process associated with $\left(\mathrm{Ch}_{n}^{r}, \mathcal{F}_{n}^{r}\right)$

$$
\mathrm{Ch}_{n}^{r}(f)=\frac{1}{2} \int_{Y_{n}^{r}}|\nabla f|_{w, Y_{n}^{r}}^{2} d m_{n, r}, \quad \mathcal{F}_{n}^{r}:=\left\{f \in L^{2}\left(Y_{n}^{r} ; m_{n, r}\right): \mathrm{Ch}_{n}^{r}(f)<\infty\right\} .
$$

Recall that $|\nabla f|_{w, Y_{n}^{r}}^{2}$ means the minimal weak upper gradient on $Y_{n}^{r}$ (see Subsection 2.4.2). We note that the Cheeger energy $\mathrm{Ch}_{n}^{r}$ on the closed ball $Y_{n}^{r}$ is also quadratic because of [6, Theorem 4.19]. Since closed balls are not necessarily convex subset in $X_{n}$, the closed ball $Y_{n}^{r}$ is not necessarily an $\operatorname{RCD}(K, \infty)$ space. However, we can still construct the Brownian motion on $Y_{n}^{r}$ since we have that $\left(\mathrm{Ch}_{n}^{r}, \mathcal{F}_{n}^{r}\right)$ is quadratic ([6, Theorem 4.19]) and $[d(x, \cdot)] \leq m_{n, r}$ ([6, (iv) Theorem 4.18]) for any fixed $x \in Y_{n}^{r}$, which imply that $\left(\mathrm{Ch}_{n}^{r}, \mathcal{F}_{n}^{r}\right)$ becomes a quasi-regular Dirichlet form by the same manner of [6, Lemma 6.7] and [9, Theorem 1.2] (see also [3, §7.2]). Here [f] means the energy measure of the Cheeger energy (see [6, (4.21)]) and $\left[d\left(\bar{x}_{n}, \cdot\right)\right] \leq m_{n, r}$ means

$$
\frac{d\left[d\left(\bar{x}_{n}, \cdot\right)\right]}{d m_{n, r}}(y) \leq 1 \quad m_{n, r} \text {-a.e. } y \in Y_{n}^{r} \text {. }
$$


Note that although [6, Lemma 6.7] assumed the $\operatorname{RCD}(K, \infty)$ condition, only the quadraticity of the Cheeger energy and $\left[d\left(\bar{x}_{n}, \cdot\right)\right] \leq m_{n, r}$ are used to construct the Brownian motions, and the $\operatorname{CD}(K, \infty)$ condition is not necessary (see also [46, §4] for more detailed studies of the Cheeger energies and Brownian motions on subsets in $\operatorname{RCD}(K, \infty)$ spaces).

We first estimate (I) ${ }_{n, \eta}$. By Lyons-Zheng decomposition ([48], and see also [27, Section 5.7]), we have

$$
h\left(B_{t}^{n}\right)-h\left(B_{s}^{n}\right)=\frac{1}{2}\left(M_{t}^{[h]}-M_{s}^{[h]}\right)+\frac{1}{2}\left(M_{T-t}^{[h]}\left(r_{T}\right)-M_{T-s}^{[h]}\left(r_{T}\right)\right), \quad \mathbb{P}_{R+r}^{m_{n}, R+r} \text {-a.e. }
$$

for $0 \leq t \leq T$.

Then by time-symmetry (see [27, Lemma 5.7.1]), we have

$$
\begin{aligned}
(\mathrm{I})_{n, \eta} \leq & \mathbb{P}_{R+r}^{m_{n, R+r}}\left(\sup _{\substack{0 \leq s, t \leq T \\
|t-s| \leq \eta}}\left|h\left(B_{t}^{n}\right)-h\left(B_{s}^{n}\right)\right|>\delta\right) \\
\leq & \mathbb{P}_{R+r}^{m_{n, R+r}}\left(\sup _{\substack{0 \leq s, t \leq T \\
|t-s| \leq \eta}}\left|M_{t}^{[h], n}-M_{s}^{[h], n}\right|>\delta\right) \\
& +\mathbb{P}_{R+r}^{m_{n, R+r}}\left(\sup _{\substack{0 \leq s, t \leq T \\
|t-s| \leq \eta}}\left|M_{T-t}^{[h], n}\left(r_{T}\right)-M_{T-s}^{[h], n}\left(r_{T}\right)\right|>\delta\right) \\
= & 2 \mathbb{P}_{R+r}^{m_{n, R+r}}\left(\sup _{\substack{0 \leq s, t \leq T \\
|t-s| \leq \eta}}\left|M_{t}^{[h], n}-M_{s}^{[h], n}\right|>\delta\right) .
\end{aligned}
$$

Since $M^{[h], n}$ is a continuous martingale, by the martingale representation theorem, there exists the one-dimensional Brownian motion $\mathbf{B}^{n}(t)$ on an extended probability space $\left(\widetilde{\Omega}, \widetilde{\mathcal{M}}, \widetilde{\mathbb{P}}_{n}^{x}\right)$ whereby $M^{[h], n}$ is represented as a time-changed Brownian motion with respect to the quadratic variation $\widetilde{\mathbb{P}}_{n}^{x}$-a.s, q.e. $x \in Y_{n}^{R+r}$ (see, e.g., Ikeda-Watanabe [34, Chapter II Theorem 7.3']). That is, for q.e. $x \in Y_{n}^{R+r}$,

$$
M_{t}^{[h], n}=\mathbf{B}^{n}\left(\left\langle M^{[h], n}\right\rangle_{t}\right)=\mathbf{B}^{n}\left(\int_{0}^{t} \frac{d \mu_{\langle h\rangle}^{n}}{d m_{n}}\left(B_{u}^{n}\right) d u\right)=\mathbf{B}^{n}\left(\int_{0}^{t}|\nabla h|_{w, Y_{n}^{R+r}}^{2}\left(B_{u}^{n}\right) d u\right) \quad \widetilde{\mathbb{P}}_{n}^{x} \text {-a.s. }
$$

The last equality followed from [6, (iv) Theorem 4.18]. Since $|\nabla h|_{w, Y_{n}^{R+r}} \leq \operatorname{Lip}(h)$, we have

$$
\begin{aligned}
& \left\{\omega \in \widetilde{\Omega}: \sup _{\substack{0 \leq s, t \leq T \\
|t-s| \leq \eta}}\left|M_{t}^{[h], n}-M_{s}^{[h], n}\right|>\delta\right\} \\
& =\left\{\omega \in \widetilde{\Omega}: \sup _{\substack{0 \leq s, t \leq T \\
|t-s| \leq \eta}}\left|\mathbf{B}^{n}\left(\int_{0}^{t}|\nabla h|_{w, Y_{n}^{R+r}}^{2}\left(B_{u}^{n}\right) d u\right)-\mathbf{B}^{n}\left(\int_{0}^{s}|\nabla h|_{w, Y_{n}^{R+r}}^{2}\left(B_{u}^{n}\right) d u\right)\right|>\delta\right\} \\
& \subset\left\{\omega \in \widetilde{\Omega}: \sup _{\substack{0 \leq s, t \leq \operatorname{Lip}(h)^{2} T \\
|t-s| \leq \operatorname{Lip}(h)^{2} \eta}}\left|\mathbf{B}^{n}(t)-\mathbf{B}^{n}(s)\right|>\delta\right\} .
\end{aligned}
$$

Let $\mathrm{W}$ be the standard Wiener measure on $C([0, \infty) ; \mathbb{R})$. Let

$$
\theta(\eta, h):=\mathbb{W}_{n}\left(\sup _{\substack{0 \leq s, t \leq \operatorname{Lip}(h)^{2} T \\|t-s| \leq \operatorname{Lip}(h)^{2} \eta}}|\omega(t)-\omega(s)|>\delta\right)
$$

By (5.11) and noting $\sup _{n \in \mathbb{N}} m_{n}\left(B_{R+r}\left(\bar{x}_{n}\right)\right)<\infty$ because of the weak convergence of 
$m_{n}$, we have, for any $T>0$,

$$
\begin{aligned}
(\mathrm{I})_{n, \eta} & \leq \sup _{n \in \mathbb{N}} \int_{X_{n}} L_{\eta, T}^{n, h} d m_{n, R+r} \\
& \leq \sup _{n \in \mathbb{N}} 2 \mathbb{P}_{R+r}^{m_{n, R+r}}\left(\sup _{\substack{0 \leq s, t \leq T \\
|\bar{t}-s| \leq \eta}}\left|M_{t}^{[h]}-M_{s}^{[h]}\right|>\delta\right) \\
& \leq 2 \theta(\eta, h) \sup _{n \in \mathbb{N}} m_{n}\left(B_{R+r}\left(\bar{x}_{n}\right)\right) \\
& \stackrel{\eta \rightarrow 0}{\rightarrow} 0 .
\end{aligned}
$$

We now estimate (II) $)_{n, \eta}$. We have the following estimate:

$$
\begin{aligned}
(\mathrm{II})_{n, \eta} & =\mathbb{P}^{m_{n, R}}\left(\sup _{\substack{0 \leq s, t \leq T \\
|t-s| \leq \eta}}\left|h\left(B_{t}^{n}\right)-h\left(B_{s}^{n}\right)\right|>\delta: \Lambda_{r}^{c}\right) \\
& \leq 6 m_{n}\left(B_{R+r}\left(\bar{x}_{n}\right)\right) \frac{1}{\sqrt{2 \pi}} \int_{\frac{2 r}{3 \sqrt{\operatorname{Lip}(h)^{2} T}}}^{\infty} \exp \left\{-\frac{s^{2}}{2}\right\} d s \\
& \leq c \exp \left\{\mathrm{c}_{2}(R+r)^{2}\right\} \int_{\frac{2 r}{3 \sqrt{\operatorname{Lip}(h)^{2} T}}}^{\infty} \exp \left\{-\frac{s^{2}}{2}\right\} d s . \\
& \leq c \exp \left\{\mathrm{c}_{2}(R+r)^{2}\right\} \frac{3 \sqrt{\operatorname{Lip}(h)^{2} T}}{2 r} \exp \left\{-\frac{r^{2}}{18 \operatorname{Lip}(h)^{2} T}\right\} \\
& r \rightarrow \infty 0 .
\end{aligned}
$$

Here $c>0$ is a constant independent of $n$. In the second line above, we used [27, Lemma 5.7.2], in the third line, we used the volume growth estimate (1.2) and, in the fourth line, we used the fact $\int_{x}^{\infty} \exp \left\{\frac{s^{2}}{2} d s\right\} \leq \frac{1}{x} \exp \left\{-\frac{x^{2}}{2}\right\}$. Thus, by (5.12) and (5.13), we have that, for any $R>0$,

$$
\lim _{\eta \rightarrow 0} \sup _{n \in \mathbb{N}} \int_{X_{n}} L_{\eta, T}^{n, h} \mathbf{1}_{B_{R}\left(\bar{x}_{n}\right)} d m_{n}=\lim _{\eta \rightarrow 0} \sup _{n \in \mathbb{N}}\left((\mathrm{I})_{n, \eta}+(\mathrm{II})_{n, \eta}\right)=0 .
$$

Thus we have the desired result (5.10).

We resume to prove Theorem 1.2.

Proof of Theorem 1.2. It is easy to check that conditions (i) and (ii) in Lemma 5.6 are satisfied with $\nu_{n}=\widetilde{m}_{n}$ in the both cases of $m_{n}\left(X_{n}\right)=\infty$ and $m_{n}\left(X_{n}\right)<\infty$. Thus we have shown the tightness. By using Lemma 5.5, we have completed the proof of (i) $\Longrightarrow$ (ii) in Theorem 1.2 in the case of $m_{n}\left(X_{n}\right)<\infty$. Moreover, we can check easily that the conditions in Lemma 5.4 are satisfied with $\nu_{n}=\widetilde{m}_{n}$ in the case of $m_{n}\left(X_{n}\right)=\infty$ (see [30, Remark 4.6]). Therefore, we have completed the proof of (i) $\Longrightarrow$ (ii) in Theorem 1.2 in the case of $m_{n}\left(X_{n}\right)=\infty$. We finish the proof of (i) $\Longrightarrow$ (ii) in Theorem 1.2.

\section{Proof of Theorem 1.4}

We show the following statement: for any $\varepsilon>0$,

(iii) $\geq \varepsilon$ There exist a complete separable metric space $(X, d)$ and isometric embeddings $\iota_{n}: X_{n} \rightarrow X(n \in \overline{\mathbb{N}})$ so that

$$
\left(\iota_{n}\left(B^{n}\right), \mathbb{P}_{n}^{\bar{x}_{n}}\right) \rightarrow\left(\iota_{\infty}\left(B^{\infty}\right), \mathbb{P}_{\infty}^{\bar{x}_{\infty}}\right) \quad \text { weakly } \quad \text { in } \mathcal{P}(C([\varepsilon, \infty) ; X)) .
$$


We first discuss the case of condition (A), that is, $m_{n}\left(X_{n}\right)=1$.

Since we have already shown the weak convergence of the finite-dimensional distributions under the general $\operatorname{RCD}(K, \infty)$ condition for starting points $\bar{x}_{n}$ in Lemma 5.1, it suffices to prove the tightness:

Lemma 6.1. Under condition (A), $\left\{\mathbb{B}_{n}^{\bar{x}_{n}}\right\}_{n \in \mathbb{N}}$ is tight in $\mathcal{P}(C([\varepsilon, \infty), X))$ for any $\varepsilon>0$.

Proof. In the proof of [30, Theorem 7.7], we have

$$
\sup _{n \in \mathbb{N}} \operatorname{Ent}_{m_{n}}\left(p_{n}\left(\varepsilon, \bar{x}_{n}, d y\right)\right)=\sup _{n \in \mathbb{N}} \operatorname{Ent}_{m_{n}}\left(\mu_{\varepsilon}^{n, \bar{x}_{n}}\right)<\infty,
$$

where $\mu_{\varepsilon}^{n, \bar{x}_{n}}:=\mathcal{H}_{\varepsilon}^{n} \delta_{\bar{x}_{n}}$ defined in Subsection 2.4.4. Let $\mathbb{B}_{n}^{\bar{x}_{n}}$ and $\mathbb{B}_{n}^{m_{n}}$ be restricted to the path space $C([\varepsilon, \infty), X)$. By using Markov property, we have that

$$
\frac{d \mathbb{B}_{n}^{\bar{x}_{n}}}{d \mathbb{B}_{n}^{m_{n}}}=p\left(\varepsilon, \bar{x}_{n}, B_{\varepsilon}^{n}\right) .
$$

In fact, we have that, for any Borel measurable functions $F: C([\varepsilon, \infty), X): \rightarrow \mathbb{R}$,

$$
\begin{aligned}
\mathbb{E}^{\bar{x}_{n}}\left(F\left(B_{\varepsilon+.}^{n}\right)\right) & =\mathbb{E}^{\bar{x}_{n}}\left(\mathbb{E}^{B_{\varepsilon}^{n}}(F)\right) \\
& =\int_{X_{n}} \mathbb{E}^{y}\left(F\left(B^{n}\right)\right) p_{n}\left(\varepsilon, \bar{x}_{n}, d y\right) \\
& =\int_{X_{n}} \mathbb{E}^{y}\left(F\left(B^{n}\right)\right) p_{n}\left(\varepsilon, \bar{x}_{n}, y\right) m_{n}(d y) \\
& =\mathbb{E}^{m_{n}}\left(p_{n}\left(\varepsilon, \bar{x}_{n}, B_{0}^{n}\right) F\left(B^{n}\right)\right) \\
& =\mathbb{E}^{m_{n}}\left(p_{n}\left(\varepsilon, \bar{x}_{n}, B_{\varepsilon}^{n}\right) F\left(B_{\varepsilon+.}^{n}\right)\right),
\end{aligned}
$$

whereby in the last line, we used the stationarity.

Let us denote $\operatorname{Ent}_{\nu}(\mu)=\operatorname{Ent}(\mu \mid \nu)$. By the fact that $\sup _{n \in \mathbb{N}} \operatorname{Ent}_{m_{n}}\left(p_{n}\left(\varepsilon, \bar{x}_{n}, d y\right)\right)<\infty$, we have

$$
\begin{aligned}
\sup _{n \in \mathbb{N}} \operatorname{Ent}\left(\mathbb{B}_{n}^{\bar{x}_{n}} \mid \mathbb{B}_{n}^{m_{n}}\right) & =\sup _{n \in \mathbb{N}} \int_{\Omega} p_{n}\left(\varepsilon, \bar{x}_{n}, B_{\varepsilon}^{n}\right) \log \left\{p_{n}\left(\varepsilon, \bar{x}_{n}, B_{\varepsilon}^{n}\right)\right\} d \mathbb{P}_{n}^{m_{n}} \\
& =\sup _{n \in \mathbb{N}} \int_{X_{n}} P_{\varepsilon}^{n}\left(p_{n}\left(\varepsilon, \bar{x}_{n}, \cdot\right) \log \left\{p_{n}\left(\varepsilon, \bar{x}_{n}, \cdot\right)\right\}\right) d m_{n} \\
& =\sup _{n \in \mathbb{N}} \int_{X_{n}} p_{n}\left(\varepsilon, \bar{x}_{n}, \cdot\right) \log \left\{p_{n}\left(\varepsilon, \bar{x}_{n}, \cdot\right)\right\} d m_{n} \\
& =\sup _{n \in \mathbb{N}} \operatorname{Ent}_{m_{n}}\left(p_{n}\left(\varepsilon, \bar{x}_{n}, d y\right)\right)<\infty .
\end{aligned}
$$

In the third line above, we used the invariance property of $m_{n}$ with respect to the heat semigroup $\left\{P_{t}^{n}\right\}_{t \geq 0}$ whereby

$$
\int_{X_{n}} P_{t}^{n} f d m_{n}=\int_{X_{n}} f d m_{n} .
$$

Since $\left\{\mathbb{B}_{n}^{m_{n}}\right\}_{n \in \mathbb{N}}$ is tight by Lemma 5.6, by using the tightness criterion with respect to the entropy [30, Proposition 4.1], we have the tightness of $\left\{\mathbb{B}_{n}^{\bar{x}_{n}}\right\}_{n \in \mathbb{N}}$.

Proof of Theorem 1.4 in the case of (A). By the weak convergence of the finite-dimensional distributions in Lemma 5.1, and the tightness in Lemma 6.1, we have finished the proof of Theorem 1.4 for the case (A).

Now we prove Theorem 1.4 in the case of condition (B). 
Proof of Theorem 1.4 in the case of $(\boldsymbol{B})$. By using Markov property, we have that, for any Borel measurable functions $F: C([\varepsilon, \infty), X) \rightarrow \mathbb{R}$,

$$
\begin{aligned}
\mathbb{E}^{\bar{x}_{n}}\left(F\left(B_{\varepsilon+.}^{n}\right)\right) & =\mathbb{E}^{\bar{x}_{n}}\left(\mathbb{E}^{B_{\varepsilon}^{n}}\left(F\left(B_{.}^{n}\right)\right)\right) \\
& =\int_{X_{n}} \mathbb{E}^{y}\left(F\left(B^{n}\right)\right) p_{n}\left(\varepsilon, \bar{x}_{n}, d y\right) \\
& =\mathbb{E}^{p_{n}\left(\varepsilon, \bar{x}_{n}, d y\right)}\left(F\left(B^{n}\right)\right) .
\end{aligned}
$$

By Theorem 2.5, it holds that $p_{n}\left(\varepsilon, \bar{x}_{n}, d y\right) \rightarrow p_{\infty}\left(\varepsilon, \bar{x}_{\infty}, d y\right)$ in $W_{2}$-sense and thus also in $W_{1}$-sense (see e.g., [70, Remark 6.6]). Therefore, condition (i) in Lemma 5.6 holds with $\nu_{n}=p_{n}\left(\varepsilon, \bar{x}_{n}, d y\right)$. Moreover, condition (ii) with $\nu_{n}=p_{n}\left(\varepsilon, \bar{x}_{n}, d y\right)$ in Lemma 5.6 also holds by the assumption (B). Therefore, by Lemma 5.4, and Lemma 5.6 with $\nu_{n}=p_{n}\left(\varepsilon, \bar{x}_{n}, d y\right)$, we have the desired result.

\section{Proof of Theorem 1.6}

Proof of Theorem 1.6. The goal of the proof is to show the pmG convergence of $\mathcal{X}_{n}$ to $\mathcal{X}_{\infty}$, that is, for any $f \in C_{b s}(X)$ (recall $C_{b s}(X)$ means the set of bounded continuous functions with bounded supports), we have

$$
\int_{X} f d m_{n} \rightarrow \int_{X} f d m_{\infty} \text { as } n \rightarrow \infty
$$

We first consider the case of $K>0$.

The case of $K>0$ :

Let $\lambda_{n}^{1}$ be the spectral gap of $\mathrm{Ch}_{n}$ :

$$
\lambda_{n}^{1}:=\inf \left\{\frac{\mathrm{Ch}_{n}(f)}{\|f\|_{L^{2}\left(m_{n}\right)}^{2}}: f \in \operatorname{Lip}\left(X_{n}\right) \backslash\{0\}, \int_{X_{n}} f d m_{n}=0\right\} .
$$

The following is a well-known fact (easy to obtain by using the spectral resolution):

$$
\left\|P_{t}^{n}-m_{n}(\cdot)\right\|_{2 \rightarrow 2} \leq e^{-\lambda_{n}^{1} t}, \quad \forall t>0,
$$

whereby $\|\cdot\|_{2 \rightarrow 2}$ means the operator norm from $L^{2}\left(X_{n} ; m_{n}\right)$ to $L^{2}\left(X_{n} ; m_{n}\right)$, and $m_{n}(f):=$ $\frac{1}{m_{n}\left(X_{n}\right)} \int_{X_{n}} f d m_{n}$.

By (7.1) and the assumption (1.5), we have that, for any $t>t_{*}\left(t_{*}\right.$ appeared in the assumption (1.5)),

$$
\begin{aligned}
& \left\|p_{n}\left(t, \bar{x}_{n}, \cdot\right)-\frac{\mathbf{1}}{m_{n}\left(X_{n}\right)}\right\|_{L^{2}\left(m_{n}\right)} \\
& \quad=\left\|\left(P_{s}^{n}-m_{n}(\cdot)\right) p_{n}\left(t-s, \bar{x}_{n}, \cdot\right)\right\|_{L^{2}\left(m_{n}\right)} \\
& \quad \leq e^{-\lambda_{n}^{1} s}\left\|p_{n}\left(t-s, \bar{x}_{n}, \cdot\right)\right\|_{L^{2}\left(m_{n}\right)} \\
& \quad=e^{-\lambda_{n}^{1} s}\left(p_{n}\left(2(t-s), \bar{x}_{n}, \bar{x}_{n}\right)\right)^{1 / 2} \quad\left(0<s<t, \quad t_{*}<h:=t-s\right) \\
& \quad<M^{1 / 2} e^{-\lambda_{n}^{1}(t-h)} .
\end{aligned}
$$

Since the global Poincaré inequality holds under the $\operatorname{CD}(K, \infty)$ condition with a positive $K>0$ (see e.g., [70, Theorem 30.25]), we have that there exists a positive constant $C_{P}=C_{P}(K)$ depending only on $K$ so that

$$
\inf _{n \in \mathbb{N}} \lambda_{n}>C_{P}>0 .
$$


By the condition of $K>0$, there exists a positive constant $C$ so that $\sup _{n \in \mathbb{N}} m_{n}\left(X_{n}\right)<C$ (see [65, Theorem 4.26]). Thus, by statement (iii) $\geq \varepsilon,(7.2)$ and (7.3), we have that, for any $\delta>0$,

$$
\begin{aligned}
& \left|\int_{X} f d m_{n}-\int_{X} f d m_{\infty}\right| \\
& =\mid \int_{X} f d m_{n}-m_{n}\left(X_{n}\right) \mathbb{E}_{n}^{\bar{x}_{n}}\left(f\left(B_{t}^{n}\right)\right)+m_{n}\left(X_{n}\right) \mathbb{E}_{n}^{\bar{x}_{n}}\left(f\left(B_{t}^{n}\right)\right)-m_{\infty}\left(X_{\infty}\right) \mathbb{E}_{\infty}^{\bar{x}_{\infty}}\left(f\left(B_{t}^{\infty}\right)\right) \\
& +m_{\infty}\left(X_{\infty}\right) \mathbb{E}_{\infty}^{\bar{x}_{\infty}}\left(f\left(B_{t}^{\infty}\right)\right)-\int_{X} f d m_{\infty} \\
& \leq C\left(\int_{X}\left|p_{n}\left(t, \bar{x}_{n}, y\right)-\frac{1}{m_{n}\left(X_{n}\right)}\right| f d m_{n}+\left|\mathbb{E}_{n}^{\bar{x}_{n}}\left(f\left(B_{t}^{n}\right)\right)-\mathbb{E}_{\infty}^{\bar{x}_{\infty}}\left(f\left(B_{t}^{\infty}\right)\right)\right|\right. \\
& \left.+\int_{X}\left|p_{\infty}\left(t, \bar{x}_{n}, y\right)-\frac{1}{m_{\infty}\left(X_{\infty}\right)}\right| f d m_{\infty}\right) \\
& \leq C\left(\|f\|_{L^{2}\left(m_{n}\right)}\left\|p_{n}\left(t, \bar{x}_{n}, \cdot\right)-\frac{1}{m_{n}\left(X_{n}\right)}\right\|_{L^{2}\left(m_{n}\right)}+\left|\mathbb{E}_{n}^{\bar{x}_{n}}\left(f\left(B_{t}^{n}\right)\right)-\mathbb{E}_{\infty}^{\bar{x}_{\infty}}\left(f\left(B_{t}^{\infty}\right)\right)\right|\right. \\
& \left.+\|f\|_{L^{2}\left(m_{\infty}\right)}\left\|p_{\infty}\left(t, \bar{x}_{\infty}, \cdot\right)-\frac{1}{m_{\infty}\left(X_{\infty}\right)}\right\|_{L^{2}\left(m_{\infty}\right)}\right) \\
& \leq C\left(\|f\|_{L^{2}\left(m_{n}\right)} M e^{-\lambda_{n}^{1}(t-h)}+\left|\mathbb{E}_{n}^{\bar{x}_{n}}\left(f\left(B_{t}^{n}\right)\right)-\mathbb{E}_{\infty}^{\bar{x}_{\infty}}\left(f\left(B_{t}^{\infty}\right)\right)\right|+\|f\|_{L^{2}\left(m_{\infty}\right)} M e^{-\lambda_{\infty}^{1}(t-h)}\right) \\
& \leq C\left(\|f\|_{L^{2}\left(m_{n}\right)} M e^{-C_{P}(t-h)}+\left|\mathbb{E}_{n}^{\bar{x}_{n}}\left(f\left(B_{t}^{n}\right)\right)-\mathbb{E}_{\infty}^{\bar{x}_{\infty}}\left(f\left(B_{t}^{\infty}\right)\right)\right|+\|f\|_{L^{2}\left(m_{\infty}\right)} M e^{-C_{P}(t-h)}\right) \\
& \rightarrow \delta+0+\delta \text { as } n \rightarrow \infty \text { and sufficiently large } t \text {. }
\end{aligned}
$$

Thus we finish the proof of Theorem 1.6 for the case of $K>0$.

The case of $\sup _{n \in \mathbb{N}} \operatorname{diam}\left(X_{n}\right)<D$ :

The case of $\sup _{n \in \mathbb{N}} \operatorname{diam}\left(X_{n}\right)<D$ can be proved in the same way as the case of $K>0$ since the local Poincaré inequality holds for any $\operatorname{RCD}(K, \infty)$ spaces (see [57, Theorem 1.1]). If $\sup _{n \in \mathbb{N}} \operatorname{diam}\left(X_{n}\right)<D$ holds, then the local Poincaré inequality is equivalent to the global Poincaré inequality and the proof will be the same as the case of $K>0$. Thus we finish the proof of Theorem 1.6.

\section{Proof of Theorem 1.7}

In this section, we prove Theorem 1.7. In the previous sections, we have already proved (i) $\Longleftrightarrow$ (ii) by Theorem 1.2. If $\sup _{n} \operatorname{diam}\left(X_{n}\right)<\infty$, then the implication of (iii) $\geq \varepsilon$ (or (iii) $\geq 0$ ) $\Longrightarrow$ (i) follows from Theorem 1.6. Thus we only have to show the implication (i) $\Longrightarrow$ (iii) $\geq 0$.

Proof of (i) $\Longrightarrow(\text { iii })_{\geq 0}$ in Theorem 1.7. Since we have already shown the weak convergence of the laws of finite-dimensional distributions in Lemma 5.1 for the general $\operatorname{RCD}(K, \infty)$ case, what we should prove is only the tightness of the Brownian motions on $C([0, \infty] ; X)$.

Lemma 8.1. $\left\{\mathbb{B}_{n}\right\}_{n \in \mathbb{N}}$ is tight in $\mathcal{P}(C([0, \infty), X))$.

Proof. Since $x_{n}$ converges to $x_{\infty}$ in $(X, d)$, the set of the laws of the initial distributions $\left\{B_{0}^{n}\right\}_{n \in \mathbb{N}}=\left\{\delta_{x_{n}}\right\}_{n \in \mathbb{N}}$ is clearly tight in $\mathcal{P}(X)$. Thus it suffices to show the following (see [13, Theorem 12.3]): for each $T>0$, there exist $\beta>0, C>0$ and $\theta>1$ such that, for all $n \in \mathbb{N}$

$$
\mathbb{E}^{x_{n}}\left[\widetilde{d}^{\beta}\left(B_{t}^{n}, B_{t+h}^{n}\right)\right] \leq C h^{\theta}, \quad(0 \leq t \leq T \text { and } 0 \leq h \leq 1)
$$

whereby $\widetilde{d}(x, y):=d(x, y) \wedge 1$. 
We first give a heat kernel estimate. Let $\sup _{n \in \mathbb{N}} \operatorname{diam}\left(X_{n}\right)<D$. By the generalized Bishop-Gromov inequality [22, Proposition 3.6], we have the following volume growth estimate: for any $D>0$, there exist positive constants $\nu=\nu(N, K, D)>0$ and $c=$ $c(N, K, D)>0$ such that, for all $n \in \mathbb{N}$

$$
m_{n}\left(B_{r}(x)\right) \geq c r^{2 \nu} \quad(0 \leq r \leq D) .
$$

In fact, by the generalized Bishop-Gromov inequality, we have that, for any $0<r \leq D<$ $\infty$,

$$
m_{n}\left(B_{r}(x)\right) \geq \frac{\int_{0}^{r} \Theta_{K / N}(t)^{N} d t}{\int_{0}^{D} \Theta_{K / N}(t)^{N} d t} m_{n}\left(B_{D}(x)\right) \geq c(N, K, D) \int_{0}^{r} \Theta_{K / N}(t)^{N} d t .
$$

Here $c(N, K, D)=\frac{1}{\int_{0}^{D} \Theta_{K / N}(t)^{N} d t}$. Thus we have (8.2). Combining (3.3) with (8.2), we have the following uniform upper heat kernel estimate:

$$
p_{n}(t, x, y) \leq \frac{C_{1}}{c t^{\nu}} \exp \left\{-C_{2} \frac{d_{n}(x, y)^{2}}{t}\right\}
$$

for all $x, y \in X_{n}$ and $0<t \leq D^{2}$. Here constants $C_{1}, C_{2}, c, \nu$ only depend on the given constants $N, K, D$. Note that the constant $C_{3}$ in (3.3) can be taken as zero under $\sup _{n \in \mathbb{N}} \operatorname{diam}\left(X_{n}\right)<D$ according to $[63,64]$ (note that the MCP condition is satisfied under the assumption of Theorem 1.7).

Take $\beta>0$ such that $\beta / 2-\nu>1$, and set $\theta=\beta / 2-\nu$. By the Markov property, we have

L.H.S. of (8.1)

$$
\begin{aligned}
& =\int_{X_{n} \times X_{n}} p_{n}\left(t, x_{n}, y\right) p_{n}(h, y, z) \widetilde{d}^{\beta}\left(\iota_{n}(y), \iota_{n}(z)\right) m_{n}(d y) m_{n}(d z) \\
& \leq \int_{X_{n} \times X_{n}} p_{n}\left(t, x_{n}, y\right) p_{n}(h, y, z) d^{\beta}\left(\iota_{n}(y), \iota_{n}(z)\right) m_{n}(d y) m_{n}(d z) .
\end{aligned}
$$

By the Gaussian heat kernel estimate (8.3), we have

$$
\begin{aligned}
\int_{X_{n}} & p_{n}(s, y, z) d^{\beta}\left(\iota_{n}(y), \iota_{n}(z)\right) m_{n}(d z) \\
& \leq \frac{C_{1}}{c s^{\nu}} \int_{X_{n}} \exp \left(-C_{2} \frac{d_{n}(y, z)^{2}}{s}\right) d^{\beta}\left(\iota_{n}(y), \iota_{n}(z)\right) m_{n}(d z) \\
& \leq \frac{C_{1}}{c s^{\nu}} \int_{X_{n}} \exp \left(-C_{2} \frac{d_{n}(y, z)^{2}}{s}\right) d_{n}^{\beta}(y, z) m_{n}(d z) \\
& \leq C_{1} c^{-1} C_{2}^{2 / \beta} s^{\beta / 2-\nu} m_{n}\left(X_{n}\right) \sup _{y, z \in X_{n}}\left\{\left(C_{2} \frac{d_{n}(y, z)^{2}}{s}\right)^{\beta / 2} \exp \left(-C_{2} \frac{d_{n}(y, z)^{2}}{s}\right)\right\} \\
& \leq C_{1} c^{-1} C_{2}^{2 / \beta} C_{3} M_{\beta} s^{\beta / 2-\nu} \\
& =C_{4} s^{\beta / 2-\nu},
\end{aligned}
$$

whereby $M_{\beta}:=\sup _{t \geq 0} t^{\beta / 2} \exp (-t), C_{3}=\sup _{n \in \mathbb{N}} m_{n}\left(X_{n}\right)$ and $C_{4}=C_{4}(N, K, D, \beta)=$ $C_{1} c^{-1} C_{2}^{2 / \beta} C_{3} M_{\beta}$ are constants dependent only on $N, K, D, \beta$ (independent of $n$ ). Note that, since $m_{n}$ converges weakly to $m_{\infty}$ and $m_{\infty}\left(X_{\infty}\right)<\infty$ because of $\operatorname{diam}\left(X_{\infty}\right)<D$, we have that $\sup _{n \in \mathbb{N}} m_{n}\left(X_{n}\right)=C_{3}<\infty$. By (8.5), we have

$$
\begin{aligned}
\text { R.H.S. of (8.4) } & \leq C_{4} h^{\beta / 2-\nu} \int_{X_{n}} p_{n}\left(t, x_{n}, y\right) m_{n}(d y) \\
& \leq C_{4} h^{\beta / 2-\nu} .
\end{aligned}
$$

Thus we finish the proof.

Thus we have completed the proof of Theorem 1.7. 


\section{References}

[1] Albeverio, S. and Kusuoka, S. (2012). Diffusion processes in thin tubes and theier limits on graphs. Ann. Probab. 40, no. 5, 2131-2167. MR-3025713

[2] Ambrosio, L. (2018). Calculus, heat flow and curvature-dimension bounds in metric measure spaces, Survey for ICM Plenary Talk: http://cvgmt.sns.it/paper/3779/.

[3] Ambrosio, L., Gigli, N., Mondino, A. and Rajala, T. (2015). Riemannian Ricci curvature lower bounds in metric measure spaces with $\sigma$-finite measure. Trans. Amer. Math. Soc. 367, no. 7, 4661-4701. MR-3335397

[4] Ambrosio, L., Gigli, N. and Savaré, G. (2015). Bakry-Émery curvature-dimension condition and Riemannian Ricci curvature bounds. Ann. Probab. 43, no. 1, 339-404. MR-3298475

[5] Ambrosio, L., Gigli, N. and Savaré, G. (2014). Calculus and heat flow in metric measure spaces and applications to spaces with Ricci bounds from below. Invent. Math. 195, 289-391. MR-3152751

[6] Ambrosio, L., Gigli, N. and Savaré, G. (2014). Metric measure spaces with Riemannian Ricci curvature bounded from below. Duke Math. J. 163, no. 7, 1405-1490. MR-3205729

[7] Ambrosio, L., Gigli, N. and Savaré, G. (2005). Gradient Flows in Metric Spaces and in the Space of Probability Measures. Lectures in Mathematics ETH Zürich. Birkhäuser Verlag, Basel. MR-2129498

[8] Ambrosio, L., Mondino, A. and Savaré, G. (2016). On the Bakry-Émery condition, the gradient estimates and the Local-to-Global property of $\operatorname{RCD}^{*}(K, N)$ metric measure spaces. J. Geom. Anal. 26, no. 1, 24-56. MR-3441502

[9] Ambrosio, L., Savaré, G. and Zambotti, L. (2009). Existence and stability for Fokker-Planck equations with log-concave reference measure. Probab. Theory Related Fields 145, 517-564. MR-2529438

[10] Athreya, S., Löhr, W. and Winter, A. (2017). Invariance principle for variable speed random walks on trees. Ann. Probab. 45, no. 2, 625-667. MR-3630284

[11] Bacher, K. and Sturm, K.-Th. (2010). Localization and tensorization properties of the curvature-dimension condition for metric measure spaces. J. Funct. Anal. 259, no. 1, 28-56. MR-2610378

[12] Bass, R. F., Kumagai, T. and Uemura, T. (2010). Convergence of symmetric Markov chains on $\mathbb{Z}^{d}$. Probab. Theory Related Fields 148, 107-140. MR-2653223

[13] Billingsley, P. (1968). Convergence of Probability Measures, 493. John Wiley \& Sons. MR0233396

[14] Blumenthal, R. M. and Getoor, R. K. (1968). Markov Processes and Potential Theory. Academic Press, New York-London. MR-0264757

[15] Burago, D., Burago, Y. and Ivanov, S. (2001). A Course in Metric Geometry. Graduate Studies in Mathematics, 33. American Mathematical Society, Providence, RI. MR-1835418

[16] Burdzy, K. and Chen, Z.-Q. (2008). Discrete approximations to reflected Brownian motion. Ann. Probab. 36, 698-727. MR-2393994

[17] Cheeger, J. and Colding, T. H. (1997). On the structure of spaces with Ricci curvature bounded below. I. J. Differential Geom. 46, no. 3, 406-480. MR-1484888

[18] Cheeger, J. and Colding, T. H. (2000). On the structure of spaces with Ricci curvature bounded below, II. J. Differential Geom. 54, 13-35. MR-1815410

[19] Cheeger, J. and Colding, T. H. (2000). On the structure of spaces with Ricci curvature bounded below, III. J. Differential Geom. 54, 37-74. MR-1815411

[20] Chen, Z.-Q., Kim, P. and Kumagai, T. (2013). Discrete approximation of symmetric jump processes on metric measure spaces. Probab. Theory Related Fields 155, 703-749. MR3034791

[21] Croydon, D. A., Hambly, B. M. and Kumagai, T. (2017). Time-changes of stochastic processes associated with resistance forms. Electron. J. Probab. 22, no. 82, 1-41. MR-3718710 
[22] Erbar, M., Kuwada, K. and Sturm, K.-Th. (2015). On the equivalence of the entropic curvaturedimension condition and Bochner's inequality on metric measure spaces. Invent. Math. 201, no. 3, 993-1071. MR-3385639

[23] Ethier, S. N. and Kurtz, T. G. (1986). Markov Processes. John Wiley \& Sons. MR-0838085

[24] Fannjiang, A. and Komorowski, T. (1997). A martingale approach to homogenization of unbounded random flows. The Annals of Probability 25, no. 4, 1872-1894. MR-1487440

[25] Freidlin, M. I. and Wentzell, A. D. (1993). Diffusion processes on graphs and the averaging principle. Ann. Probab. 21, 2215-2245. MR-1245308

[26] Freidlin, M. I. and Wentzell, A. D. (2004). Diffusion processes on an open book and the averaging principle. Stochastic Process. Appl. 113, 101-126. MR-2078539

[27] Fukushima, M., Oshima, Y. and Takeda, M. (2011). Dirichlet Forms and Symmetric Markov Processes. 2nd revised and extended edition. Study in Mathematics, 19. De Gruyter. MR2778606

[28] Galaz-García, F., Kell, M., Mondino, A. and Sosa, G. (2018). On quotient of spaces with Ricci curvature bounded below, preprint, J. Func. Anal. 275, no. 6, 1368-1446. MR-3820328

[29] Gigli, N. (2015). On the differential structure of metric measure spaces and applications. Mem. Amer. Math. Soc. 236, no. 1113. MR-3381131

[30] Gigli, N., Mondino, A. and Savaré, G. (2015). Convergence of pointed non-compact metric measure spaces and stability of Ricci curvature bounds and heat flows. Proc. Lond. Math. Soc. (3) 111, no. 5, 1071-1129. MR-3477230

[31] Gordina, M. and Laetsch, T. (2017). Weak convergence to Brownian motion on sub-Riemannian manifolds. Trans. Amer. Math. Soc. 369, 6263-6278. MR-3660220

[32] Gromov, M. (1999). Metric Structures for Riemannian and Non-Riemannian Spaces, Progress in Mathematics, 152. Birkhäuser Boston Inc., Boston, MA. Based on the 1981 French original. With appendices by M. Katz, P. Pansu and S. Semmes. Translated from the French by S. M. Bates. MR-1699320

[33] Ikeda, N. and Ogura, Y. (1990). A degenerating sequence of Riemannian metrics on a manifold and their Brownian motions. Diffusion Processes and Related Problems in Analysis, I: ed. by M. A. Pinsky. Birkhäuser. 293-312. MR-1110170

[34] Ikeda, N. and Watanabe, S. (1989). Stochastic Differential Equations and Diffusion Processes. North-Holland and Kodan-sha, Amsterdam and Tokyo. MR-0637061

[35] Jiang, R., Li, H. and Zhang, H. (2016). Heat kernel bounds on metric measure spaces and some applications. Potential Anal. 44, 601-627. MR-3489857

[36] Kasue, A. and Kumura, H. (1994). Spectral convergence of Riemannian manifolds. Tôhoku Math. J. 46, 147-179. MR-1272877

[37] Kasue, A. and Kumura, H. (1996). Spectral convergence of Riemannian manifolds II. Tôhoku Math. J. 48, 71-120. MR-1373175

[38] Kavian, O., Kerkyacharian, G. and Roynette, B. (1993). Quelques remarques sur l'ultracontractivité. J. Funct. Anal. 111, no. 1, 155-196. MR-1200640

[39] Ketterer, C. (2013). Ricci curvature bounds for warped products. Journal of Functional Analysis 265, no. 2, 266-299. MR-3056704

[40] Ketterer, C. (2015). Cones over metric measure spaces and the maximal diameter theorem. $J$. Math. Pures Appl. (9) 103, no. 5, 1228-1275. MR-3333056

[41] Kumagai, T. (2014). Random Walks on Disordered Media and their Scaling Limits. Lecture Notes in Mathematics, 2101, École d'Été de Probabilités de Saint-Flour XL-2010. Springer, New York. MR-3156983

[42] Kuwae, K., Machigashira, Y. and Shioya, T. (2001). Sobolev spaces, Laplacian, and heat kernel on Alexandrov spaces. Math. Z. 238, no. 2, 269-316. MR-1865418

[43] Kuwae, K. and Shioya, S. (2003). Convergence of spectral structures: a functional analytic theory and its applications to spectral geometry. Commun. Anal. Geom. 11, 599-673. MR2015170

[44] Li, X.-M. (2016). Limits of random differential equations on manifolds. Probability Theory and Related Fields 166, no. 3-4, 659-712. MR-3568037 
[45] Li, X.-M. (2017). Homogenisation on homogeneous spaces (with an appendix by D. Rumynin). To appear in Journal of the Mathematical Society of Japan. MR-3787732

[46] Lierl, J. and Sturm, K.-Th. (2018). Neumann heat flow and gradient flow for the entropy on non-convex domains. Calc. Var. and PDE's 57, 25. MR-3742815

[47] Lott, J. and Villani, C. (2009). Ricci curvature for metric-measure spaces via optimal transport. Ann. of Math. (2) 169, 903-991. MR-2480619

[48] Lyons, T. J. and Zhang, T. S. (1994). Decomposition of Dirichlet processes and its applications. textitAnn. Prob. 22, no. 1, 494-524. 157-158, 249-272. MR-1258888

[49] Ma, Z.-M. and Röckner, M. (1992). Introduction to the Theory of (Nonsymmetric) Dirichlet Forms. Universitext. Springer, Berlin. MR-1214375

[50] McShane, E. J. (1934). Extension of range of functions, Bull. Amer. Math. Soc. 40, no. 12, 837-842. MR-1562984

[51] Ogura, Y. (2001). Weak convergence of laws of stochastic processes on Riemannian manifolds. Probab. Theory Related Fields 119 529-557. MR-1826406

[52] Ogura, Y., Tomisaki, M. and Tsuchiya, M. (2002). Convergence of local type Dirichlet forms to a non-local type one. Ann. I. H. Poincaré-PR 38, no. 4, 507-556. MR-1914938

[53] Otsu, Y. and Shioya, T. (1994). The Riemannian structure of Alexandorff spaces. J. Differential Geometry 39, 629-658. MR-1274133

[54] Petrunin, A. (2007). Semiconcave functions in Alexandrov's geometry. In Surveys in Differential Geometry 11, 137-201. Int. Press, Somerville, MA. MR-2408266

[55] Petrunin, A. (2011). Alexandrov meets Lott-Villani-Sturm. Münster J. Math. 4, 53-64. MR2869253

[56] Pinsky, M. A. (1976). Isotropic transport process on a Riemannian manifold. Trans. Amer. Math. Soc. 218, 353-360. MR-0402957

[57] Rajala, T. (2012). Local Poincaré inequalities from stable curvature conditions on metric spaces. Calc. Var. Partial Differential Equations. 44, no. 3-4, 477-494. MR-2915330

[58] Rajala, T. and Sturm, K. T. (2014). Non-branching geodesics and optimal maps in strong $\mathrm{CD}(K, \infty)$-spaces. Calc. Var. PDEs 50, no. 3-4, 831-846. MR-3216835

[59] von Renesse, M.-K. and Sturm, K. T. (2005). Transport inequalities, gradient estimates, entropy and Ricci curvature. Comm. Pure Appl. Math. 68, 923-940. MR-2142879

[60] Stroock, D. W. and Varadhan, S. R. S. (1979). Multidimensional Diffusion Processes. SpringerVerlag, Berlin. MR-0532498

[61] Stroock, D. W. and Zheng, W. (1997). Markov chain approximations to symmetric diffusions. Ann. Inst. Henri Poincaré Probab. Stat. 33, 619-649. MR-1473568

[62] Sturm, K.-Th. (1994). Analysis on local Dirichlet spaces. I. Recurrence, conservativeness and $L^{p}$ - Liouville properties. J. Reine Angew. Math. 456, 173-196. MR-1301456

[63] Sturm, K.-Th. (1996). Analysis on local Dirichlet spaces. III. The parabolic Harnack inequality. J. Math. Pures. Appl. (9) 75, no. 3, 273-297. MR-1387522

[64] Sturm, K.-Th. (1998). Diffusion processes and heat kernels on metric spaces. Ann. Probab. 26, 1-55. MR-1617040

[65] Sturm, K.-Th. (2006). On the geometry of metric measure spaces. Acta Math. 196, 65-131. MR-2237206

[66] Sturm, K.-Th. (2006). On the geometry of metric measure spaces. II. Acta Math. 196, 133-177. MR-2237207

[67] Suzuki, K. (2014). Convergences and projection Markov property of Markov processes on ultrametric spaces. Publ. Res. Inst. Math. Sci. 50, no. 3, 569-588. MR-3262449

[68] Suzuki, K. Convergence of continuous stochastic processes on compact metric spaces converging in the Lipschitz distance. Potential Analysis. Online First Published in January 2018. MR-3905528

[69] Suzuki, K. (2018). Convergence of non-symmetric diffusion processes on RCD spaces. Calc. Var. and PDE's 57, 120. MR-3831291 
[70] Villani, C. (2009). Optimal Transport, Old and New. Springer-Verlag, Berlin-Heidelberg. MR-2459454

[71] Zhang, H.-C. and Zhu, X.-P. (2010). Ricci curvature on Alexandrov spaces and rigidity theorems. Comm. Anal. Geom. 18, no. 3, 503-553. MR-2747437

Acknowledgments. The author appreciates Prof. Takashi Kumagai for suggesting a constructive comment for the proof in Theorem 1.4 in the case of (B). He also expresses his great appreciation to Prof. Shouhei Honda and Prof. Yu Kitabeppu for giving a lot of valuable comments about $\operatorname{RCD}^{*}(K, N)$ spaces and sparing much time to discuss. He also appreciates Dr. Han Bangxian for fruitful discussions about Theorem 1.6. He is indebted to Mrs. Anna Katharina Suzuki-Klasen for her attentive proofreading with helpful suggestions and constructive comments. Finally, he appreciates the anonymous referee for making several corrections. This work was supported by Grant-in-Aid for JSPS Fellows Number 261798 and also supported by University Grants for student exchange between universities in partnership of Kyoto University and Hausdorff Center for Mathematics in Bonn. 